\title{
Origami lattices and folding-induced lattice transformations
}

\author{
Hongbin Fang $\odot,{ }^{1,2, *}$ Suyi $\mathrm{Li}^{3}{ }^{3}$ Manoj Thota, ${ }^{2}$ and K. W. Wang ${ }^{2}$ \\ ${ }^{1}$ Institute of AI and Robotics, Fudan University, Shanghai 200433, China \\ ${ }^{2}$ Department of Mechanical Engineering, University of Michigan, Ann Arbor, Michigan 48109, USA \\ ${ }^{3}$ Department of Mechanical Engineering, Clemson University, Clemson, South Carolina 29634, USA
}

(Received 20 January 2019; published 9 September 2019)

\begin{abstract}
Lattices and their underlying symmetries play a central role in determining the physical properties and applications of many natural and engineered materials. This research offers a comprehensive solution to a long-standing challenge regarding the lattice-based materials: how to systematically construct a lattice and transform it among different symmetric configurations in a predictable, scalable, and reversible way. By bridging the lattice geometry and rigid-folding kinematics of a group of origamis consisting of generic degree-4 vertices, we successfully construct all types of two- and three-dimensional Bravais lattices, and demonstrate that they can undergo all diffusionless phase transformations via rigid folding (i.e., dilation, extension, contraction, shear, and shuffle). Such folding-induced lattice transformations can trigger fundamental lattice-symmetry switches, which can either maintain or reconstruct the nearest-neighbor relationships according to a continuous symmetry measure. This study can foster the next generation of transformable lattice structures and materials with on-demand property tuning capabilities.
\end{abstract}

DOI: 10.1103/PhysRevResearch.1.023010

\section{INTRODUCTION}

Lattices and their underlying symmetries play a central role in determining physical properties-such as band structure, compressibility, and elastic modulus - of many natural $[1,2]$ and engineered materials [3,4]. Such a lattice-property relationship is particularly evident in metamaterials [5-7]. Therefore, purposefully designing and adjusting lattice topology can significantly expand the achievable material property range [8-10]. However, due to the lack of a flexible and versatile platform, constructing lattice structures from the ground up is challenging, and once the material is synthesized, its constituent lattice typically cannot be modified. Several studies have been proposed for tuning the underlying lattice structures of metamaterials; however, they mainly stay at a case study level, and the range and freedom of lattice adjustment are limited [11-13]. Therefore, there is a lack of an integrated and scalable approach for reconfiguring lattice structures on demand, let alone transforming their symmetry properties. Here we offer a solution to fill this gap by exploring and exploiting origami folding. The research will answer the long-standing question of how to systematically construct a lattice and transform it among different symmetric

\footnotetext{
*Present address: Institute of AI and Robotics, Fudan University, 220 Handan Rd., Yangpu District, Shanghai 200433, China; fanghongbin@fudan.edu.cn

Published by the American Physical Society under the terms of the Creative Commons Attribution 4.0 International license. Further distribution of this work must maintain attribution to the author(s) and the published article's title, journal citation, and DOI.
}

configurations in a predictable, scalable, and reversible way. Due to the broad importance of lattice structure and its transformations, answers to this question will be extremely valuable in many fields including lattice and symmetry theory, metamaterials, adaptive materials and structures, and origami science itself.

Origami has become a popular subject among mathematicians, educators, physicists, and engineers owing to the seemingly infinite possibilities of transforming two-dimensional (2D) sheets into three-dimensional (3D) shapes via folding [14-18]. Historically, such folding-induced shape transformations have been examined based on the spatial positions and orientations of its facet surfaces and crease lines [19,20]. For example, many origami-based mechanical metamaterials are analyzed by considering the folding as coordinated facet rotations with respect to the hingelike creases-essentially a linkage mechanism [21,22]. In this study, we examine the origami folding through a different lens by asking how folding can spatially arrange and rearrange the characteristic entities in the origami. These characteristic entities can be the vertices where crease lines intersect, or the center points of crease lines and facets. By treating these entities as the elements of a lattice (aka lattice points), we uncover that origami offers a remarkably comprehensive framework to construct all 2D and 3D Bravais lattices, induce all types of diffusionless lattice transformations (aka dilation, extension, contraction, shear, and shuffle), switch the lattice structure among different symmetric configurations, and even reconstruct the nearest-neighbor relationships based on a continuous symmetry measure. Therefore, this result can fundamentally advance our capability to engineer high-performance material systems by incorporating the rich design and kinematics of origami folding. 


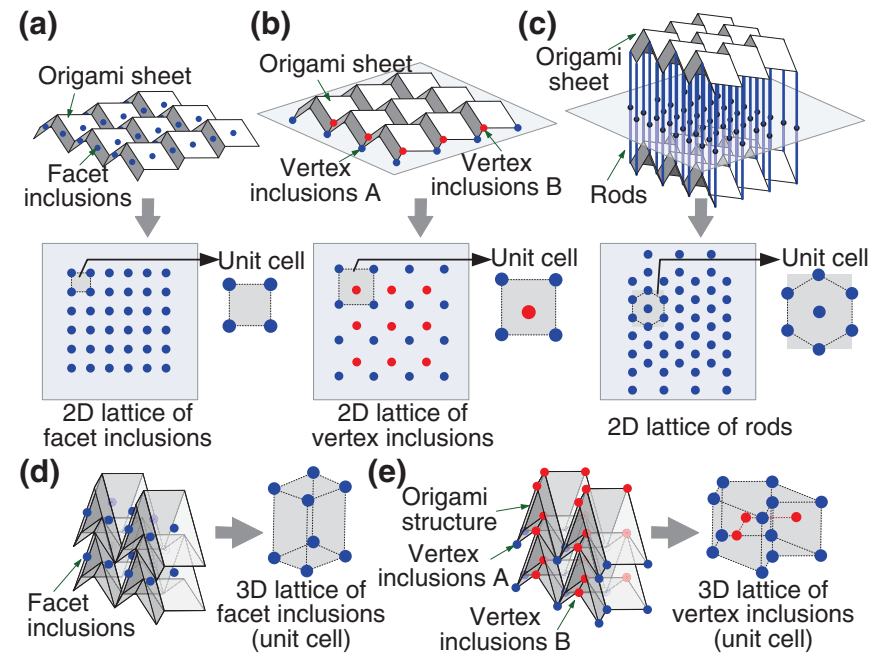

FIG. 1. Construction of origami lattices of inclusions. As examples, 2D lattices of (a) facet inclusions (square lattice), (b) vertex inclusions (non-Bravais lattice), and (c) rods (hexagonal lattice) are generated based on Miura-ori sheets; 3D lattices of (d) facet inclusions (primitive orthorhombic lattice) and (e) vertex inclusions (nonBravais lattice) are generated based on stacked Miura-ori structures. Unit cells of these lattices are highlighted.

\section{RESULTS}

\section{A. Origami lattice constructions}

Origami structures are versatile scaffolds for constructing 2D and 3D lattices. Here we selectively assign lattice points either at the facet centers [Figs. 1(a) and 1(d)] or vertices [Figs. 1(b) and 1(e)]. These lattice points can be occupied by inclusions of the same type [Figs. 1(a), 1(c), and 1(d)] or different types [Figs. 1(b) and 1(e)]. The inclusions can be selected according to targeted applications, such as conducting element [23] and sonic barrier rods [24,25]. Assembly of multiple origami sheets can further enrich the $3 \mathrm{D}$ design space [Figs. 1(c)-1(e)]. By establishing correlations between the origami geometry and the lattice configuration (see details in Appendix A for 2D Bravais lattices, and Appendix B for 3D Bravais lattices), we discover that all five types of 2D and 14 types of 3D Bravais lattices - a well-established description of the lattice configuration according to symmetry-can be constructed on a group of simple and rigid-foldable origamis consisting of generic degree-4 vertices [26,27]. The mapping between origami geometry and lattice configuration is not one-to-one in that multiple origami designs and lattice-point assignments can satisfy the reflection and rotational symmetry requirements of one specific Bravais lattice. Such a property is beneficial to lattice construction in that it provides abundant candidate origami patterns as well as enough flexibility to arrange lattice points. For a specific Bravais lattice, the symmetry requirements, as well as the constraints on axial distances and axial angles (Appendixes A and B), could be used as guidelines for origami design and lattice-point assignment. Once they are prescribed, the corresponding lattice at any intermediate state during folding could be uniquely determined.
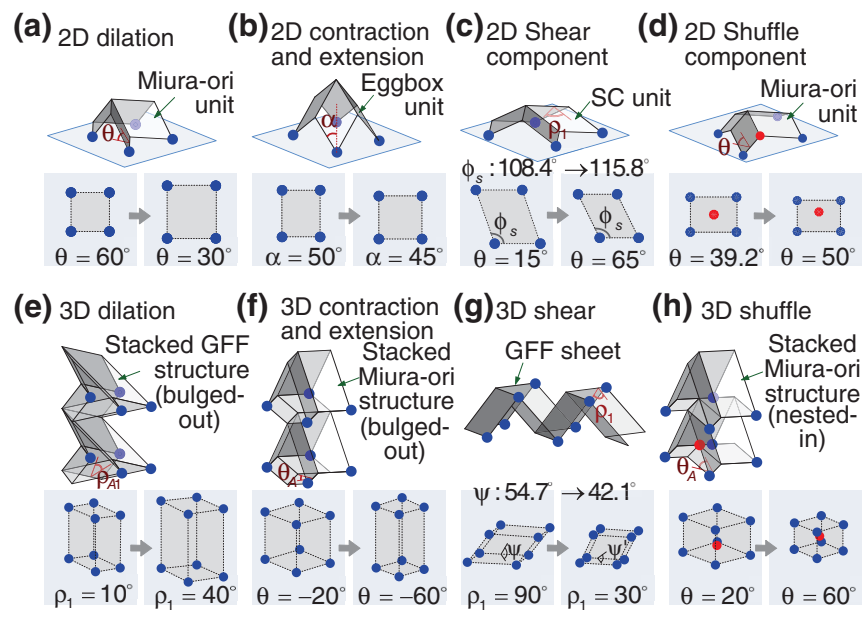

FIG. 2. Diffusionless phase transformations in origami lattices. Folding of origami structures and the corresponding lattice transformations for (a) 2D dilation, (b) 2D contraction and extension, (c) 2D shear component, (d) 2D shuffle component, (e) 3D dilation, (f) 3D contraction and extension, (g) 3D shear component, and (h) $3 \mathrm{D}$ shuffle component. Folding is represented as the change of certain geometric angle. Detailed geometries and kinematics of these origami structures can be found in Appendix C.

\section{B. Folding-induced diffusionless phase transformation and discrete symmetry switches}

Besides being a versatile platform for lattice construction, origami also provides effective mechanisms to transform these lattices via folding. By translating the folding-induced facet and vertex rearrangement into the corresponding lattice-point movements (Fig. 2), we discover that folding can impart all kinds of diffusionless phase transformations via attaining both lattice-distortive strains and shuffles. The former further includes dilation, contraction and extension, and shear [28]. Appendix $\mathrm{C}$ thoroughly examines the mathematical correspondence between origami folding and 2D and 3D lattice transformations. For each type of lattice transforamtion, a transformation matrix is formulated to quantitatively describe the changes of lattice vectors during folding. Particularly, correlations between origami's kinematic properties and lattice transformations are also established. For 2D lattices of vertex inclusions, we show that origami sheets with a negative in-plane Poisson's ratio (e.g., Miura-ori [22]) can induce 2D dilations [Fig. 2(a)]; those with a positive inplane Poisson's ratio (e.g., eggbox pattern [29]) can bring about 2D contractions and extensions [Fig. 2(b)]; and those with in-plane shear deformations (e.g., single-collinear (SC) origami [27]) can trigger 2D shear components [Fig. 2(c)]. Two-dimensional shuffle, manifested as a small movement of a lattice point (red) within the unit cell, can also be achieved in a Miura-ori-based lattice [Fig. 2(d)]. For 3D lattices of vertex inclusions, 3D dilation is achievable by exploiting the tridirectional auxeticity of a stacked general flat-foldable (GFF) structure [27] [Fig. 2(e)]; 3D contraction and extension is a result of the combination of positive and negative Poisson's ratios in a stacked Miura-ori structure [22] [Fig. 2(f)]; 3D shear is achievable due to the out-of-plane shear deformation in a GFF sheet [Fig. 2(g)] [27]; and 3D 
shuffle is evident when the lattice point locating at the center vertex of the Miura-ori cell translates within the 3D unit lattice cell during folding [Fig. 2(h)]. It is worth noting that the shear or shuffle components do not occur alone, and they are always accompanied by dilation or contraction and extension. These rich connections between origami kinematics and lattice-point movements can enable us to program a broad range of lattice transformations - according to the Cohen's classification, for example [30] - by purposefully designing the crease patterns and their folding motions.

More importantly, these folding-induced diffusionless transformations can fundamentally switch the lattices' underlying symmetry groups. For instance, the $2 \mathrm{D}$ contraction and extension could add an additional reflection symmetry to the rectangular lattice based on Miura-ori and switch it into a square lattice [i.e., the symmetry group switches from $D_{2}$ to $D_{4}$, Fig. 2(b)]. The 2D shuffle, on the other hand, could break down a reflection symmetry of a centered rectangular lattice and evolve it into a non-Bravais lattice $\left[D_{2}\right.$ to $D_{1}$, Fig. 2(d)]. Remarkably, with a carefully designed crease pattern, origami folding can switch the corresponding lattice among more than two Bravais types, which could appear either alternatively or repeatedly. Taking the Miura-ori pattern as an example, by prescribing the crease design parameters $(a, c$, and $\gamma$ ), the corresponding 2D lattices of rod inclusions could reach four types of 2D Bravais lattice: rectangular (R), centered rectangular (CR), square $(\mathrm{S})$, and hexagonal $(\mathrm{H})$ lattices [24]. For instance, if $c / a=1$ and $\gamma=60^{\circ}$, the Miura-ori-based lattice of rod inclusions will undergo a series of discrete switches: from being a hexagonal lattice $\left(\theta=0^{\circ}\right)$ to a square lattice $\left(\theta=54.7^{\circ}\right)$, to a hexagonal lattice again $\left(\theta=70.5^{\circ}\right)$, and finally to a rectangular lattice $\left(\theta \rightarrow 90^{\circ}\right)$ [Fig. 3(a)]. Note that between these switches, the origami lattices are non-Bravais, which are not denoted in Fig. 3(a). Detailed parametric analysis and another example of discrete symmetry switches can be found in the Supplemental Material, S1 [31].

\section{Folding-induced continuous symmetry evolutions in origami lattices}

So far, we have been focusing on discrete folding configurations where the corresponding lattices are strictly symmetric. However, folding is a continuous process; when the origami is folded slightly away from these strictly symmetric configurations, the corresponding lattice no longer possesses certain symmetry in a strict sense but is still very close. Therefore, it is necessary to derive a quantitative method to analyze the folding-induced and continuous changes in the lattice symmetry. Here we adopt the concept of continuous symmetry measure (CSM), which was first proposed in the field of structural chemistry [32,33]. Given a shape and a symmetry group $G$ (such as $D_{2}$ and $D_{4}$ ), the continuous symmetry measure, $S(G)$, quantifies the minimal displacement that the points of the object have to undergo in order to be transformed into a shape with $G$ symmetry. Hence, an object features a zero $S(G)$ when it is strictly $G$ symmetric; if this object has to undergo larger displacements to acquire $G$ symmetry, its $S(G)$ value increases accordingly. In other words, rather than a "black and white" discrete approach to describe symmetry (that is, either symmetric or asymmetric), the CSM offers a
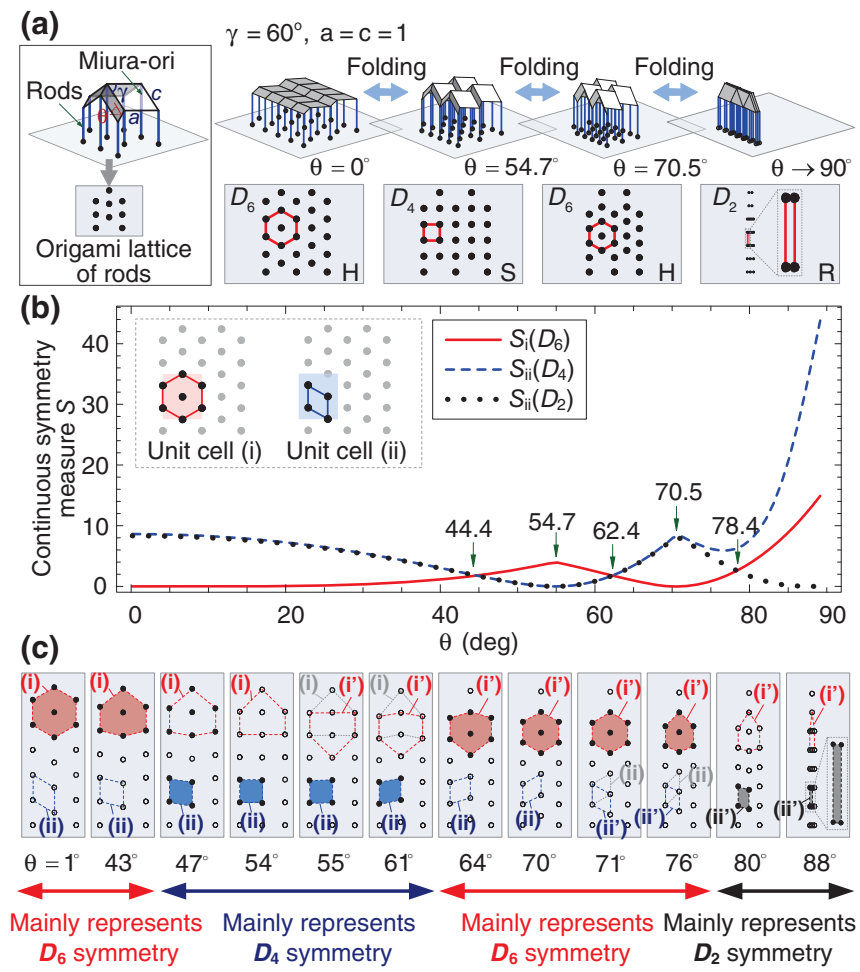

FIG. 3. Discrete and continuous symmetry measures as well as the reconstruction of the nearest-neighbor relationship of the origami lattices. Based on a Miura-ori sheet with $c / a=1, \gamma=60^{\circ}$, (a) demonstrates the discrete switches of the lattice from a hexagonal, to a square, to another hexagonal, and finally to a rectangular type. Correspondingly, the symmetry switches from $D_{6}$, to $D_{4}$, to $D_{6}$, and finally to $D_{2}$. Based on the same design, (b) displays the CSM values of the two unit cells (i) and (ii) with respect to symmetry groups $D_{6}, D_{4}$, and $D_{2}$, where the arrows denote the folding angles when reconstructions of the nearest-neighbor relationship happen. For clear illustration, lattices at specific folding configurations are provided in (c). The unit cell (i) deforms via weak transformations when $\theta<54.7^{\circ}$; as the folding reaches and passes $\theta=54.7^{\circ}$, a reconstructive transformation occurs and a new unit cell (i') with reconstructed nearest lattice-point neighbors is generated. Similar reconstructive transformation happens on unit cell (ii) when the folding reaches and passes $\theta=70.5^{\circ}$, with a new unit cell (ii') being generated. In examining the overall lattice, we observe another type of reconstructive transformation. When reaching and passing $\theta=44.4^{\circ}$, the nearest neighbors change from those lattice points constituting the unit (i) to those constituting the unit cell (ii); the lattice mainly represents $D_{6}$ and $D_{4}$ symmetry before and after $\theta=$ $44.4^{\circ}$, respectively. Similar reconstructions also occur at $\theta=62.4^{\circ}$ and $78.4^{\circ}$. At each folding configuration, the lattice with the nearest neighbors is denoted by a shaded polygon with solid lattice points.

more continuous "gray" scale to characterize the strength of a particular type of symmetry group. Detailed mathematical description of this concept and its application in quantifying origami lattice can be found in the Supplemental Material, S2 [31], and in [32,34]. By adopting this measure, we are able to describe how much the origami lattice possesses certain symmetry and understand its nearest symmetric correspondence at a certain folding configuration. 
As an example, we revisit the case in Fig. 3(a) by evaluating the CSM of the origami lattice with respect to symmetry groups $D_{6}, D_{4}$, and $D_{2}$, which correspond to the hexagonal, square, and rectangular (or centered rectangular) lattices, respectively. We select a hexagon unit cell (i) and a parallelogram unit cell (ii) of the lattice and calculate their CSM values with respect to different symmetry groups throughout the folding range from $\theta=0^{\circ}$ (fully flat) to $\theta=90^{\circ}$ (fully folded) (Fig. 3(b) (see detailed calculation procedures in the Supplemental Material, S2 [31]). At $\theta=0^{\circ}, S_{\mathrm{i}}\left(D_{6}\right)=0$ for cell (i), meaning that the lattice is of hexagonal type and strictly exhibits $D_{6}$ symmetry. As $\theta$ increases, $S_{\mathrm{i}}\left(D_{6}\right)$ gradually increases, indicating a loss of $D_{4}$ symmetry; however, $S_{\text {ii }}\left(D_{4}\right)$ decreases for cell (ii), which is a signal of strengthening $D_{6}$ symmetry. At $\theta=54.7^{\circ}, S_{\mathrm{i}}\left(D_{6}\right)$ reaches a local maximum whereas $S_{\mathrm{ii}}\left(D_{4}\right)=0$; this suggests that the lattice is far away from $D_{6}$ symmetry but fully acquires the $D_{4}$ symmetry (aka square lattice). As $\theta$ further increases, $S_{\mathrm{i}}\left(D_{6}\right)$ starts to decrease but $S_{\mathrm{ii}}\left(D_{4}\right)$ increases. At $\theta=70.5^{\circ}, S_{\mathrm{i}}\left(D_{6}\right)$ returns to zero while $S_{\mathrm{ii}}\left(D_{4}\right)$ reaches a local maximum, implying that the lattice fully regains $D_{6}$ and loses $D_{4}$ symmetry. At the final stage of folding, $S_{\mathrm{ii}}\left(D_{2}\right)$ converges to zero while $S_{\mathrm{ii}}\left(D_{4}\right)$ climbs quickly, indicating the emergence of a rectangular lattice with $D_{2}$ symmetry when $\theta$ approaches $90^{\circ}$.

\section{Breaking and reconstructing of the nearest-neighbor relationship in origami lattices}

To understand the physics underpinning the foldinginduced discrete symmetry switches and CSM evolutions, we carefully examine the lattice-point movements during these transformations. Around those configurations with strict symmetry, even with a relatively small range of folding, the latticepoint movements can be sufficient to switch the symmetry group but not break the nearest-neighbor relationships. For example, the contraction and extension shown in Fig. 2(b) can switch the symmetry group from $D_{2}$ to $D_{4}$ by slightly moving the four lattice points in the unit cell such that the corresponding rectangle changes to a square, but the unit cell always consists of the same four lattice points during this process. Such a transformation mechanism that maintains the nearest-neighbor relationship is defined as weak, which is manifested as smooth variations of the CSM value in Fig. 3(b). However, near some critical folding configurations, the lattice points within the unit cell move in a way that they are no longer the nearest neighbors, so that a new unit cell of the same type is generated by incorporating a different set of lattice points. Such a mechanism involving breaking and reconstructing of nearest-neighbor relationships is defined as reconstructive. ${ }^{1}$ For example, in Fig. 3(c), the hexagonal shape formed by the lattice points of unit cell (i) first undergoes a weak transformation, from $\theta=0^{\circ}$ to near $54.7^{\circ}$, as it becomes a houselike shape. When $\theta$ reaches and passes $54.7^{\circ}$, the original nearest-neighbor relationship cannot be maintained.

\footnotetext{
${ }^{1}$ It is worth noting that here "reconstructive" does not mean "diffusional" but rather denotes the breaking and regeneration of the nearest-neighbor relationship; all transformations discussed in this paper are diffusionless.
}

Specifically, one of the lattice points in unit cell (i) is replaced by a new lattice point such that a new unit cell (i') with the nearest lattice-point neighbors is reconstructed. In the CSM plot, such reconstructive transformation is manifested as the nonsmooth maxima corresponding to this particular unit cell (e.g., the maxima at $\theta=54.7^{\circ}$ for unit cell (i) and the maxima at $\theta=70.5^{\circ}$ for unit cell (ii)).

The reconstructive mechanisms discussed so far are defined on a particular type of unit cell, and we can extend its definition by considering different types of unit cells in the origami lattice simultaneously. At any folding configurations, the unit cell with a relatively lower value of the CSM better represents the prominent symmetry of the overall lattice. Therefore, among different types of unit cell, when there is a change in terms of the smallest CSM, the nearestneighbor relationship of the lattice can be also considered to experience a reconstruction. The difference here is that such a reconstruction occurs between unit cells of different types. In the example shown in Fig. 3, $S_{\mathrm{i}}\left(D_{6}\right)$ is lower than $S_{\mathrm{ii}}\left(D_{4}\right)$ before $\theta$ reaches $44.4^{\circ}$, indicating that the lattice points in the unit cell (i) constitutes the nearest-neighbor relationship of the overall lattice, and the overall lattice mainly represents $D_{6}$ symmetry; however, $S_{\mathrm{i}}\left(D_{6}\right)$ outstrips $S_{\mathrm{ii}}\left(D_{4}\right)$ as $\theta$ passes $44.4^{\circ}$, suggesting that the lattice points in the unit cell (ii) become the nearest neighbors, and the overall lattice mainly exhibits $D_{4}$ symmetry. Based on this generalized definition of the reconstructive mechanism, it is evident from Fig. 3 that folding can trigger multiple and successive reconstructions of the nearest-neighbor relationships (e.g., at $\theta=44.4^{\circ}, \theta=$ $62.4^{\circ}$, and $\theta=78.4^{\circ}$ ).

Three-dimensional Bravais lattices constructed on the generic four-vertex origami can also exhibit symmetry switches and evolutions by folding. We find that lattices of the same centering type (primitive, body-centered, basecentered, and face-centered lattices) possess the potential to evolve among different crystal families, which need further exploration. It is also worth highlighting that unlike certain martensitic transformations that are irreversible [1,2], the above origami folding-induced transformations are always reversible even after multiple reconstructions of the nearestneighbor relationship.

\section{SUMMARY AND DISCUSSIONS}

This study uncovers and elucidates the comprehensiveness of the capability to construct and reconfigure lattice structures by uniquely exploring origami folding. All five types of $2 \mathrm{D}$ and 14 types of 3D Bravais lattices can be constructed by exploiting the origami design space and by carefully selecting its characteristic points for lattice-point assignment. All 2D and 3D diffusionless lattice transformations-including dilation, extension and contraction, shear, and shuffle-can be achieved in such origami lattices via rigid folding. More importantly, we discover that folding can induce continuous and reversible evolutions of the lattice symmetry, and these symmetry evolutions can either maintain or reconstruct the nearest-neighbor relationship in a predictable manner.

Since the lattice structure and its symmetry properties directly govern many physical properties, the origami lattice construction and transformation offers us great freedom to 
architect programmable and adaptive metamaterials. Here the lattice points can be occupied by any components used for metamaterials, including geometric entities $[9,35]$, mechanical units [36], acoustic modules [37], electromagnetic devices [7,23], and photonic elements [7,38], etc. Attaching these components to the origami scaffold gives us metamaterials and metastructures (from nanometer-scale DNA origami [39] to meter-scale origami space structure [16]) whose mechanical, thermal, acoustic, optical, or electromagnetic properties can be effectively tailored by folding on demand. For example, our previous research demonstrated how embedding cylindrical rods to a Miura-ori sheet can result in a transformable lattice $[24,25,40]$. Folding, in this case, can effectively tune the lattice symmetry and the lattice axial lengths and significantly change the sound wave propagation behavior in terms of band gaps (see a detailed example in the Supplemental Material, S3 [31]). Such an origami lattice of rod inclusions could be used as sonic barriers for noise mitigation. Moreover, in addition to the global lattice transformation via folding, perturbing the positions of specific lattice points can introduce local defects (e.g., pop-through defects [41]) into the origami lattice, which may impart additional property programmability to the lattice-based metamaterials or metastructures.

Finally, it is worth emphasizing that, unlike the application case studies in [24,25], this research is unique and significant in that it uncovers a fundamental and comprehensive theoretical framework on the correlations between origami geometries and lattice configurations. By establishing origami as a versatile scaffold to construct and transform lattices with continuous symmetry evolutions, this theoretical framework can open the door to engineering metamaterials and metastructures that can alter their underlying lattice structures on demand. The results of this research can serve as design guidelines and analytical tools for future studies on the engineering of lattice control via origami scaffolds of different size scales, for many other applications.

\section{ACKNOWLEDGMENTS}

The authors would like to thank Narayanan Kidambi for the helpful discussions. H.F. acknowledges the support from the National Natural Science Foundation of China (Awards No. 11902078 and No. 91748203). H.F., M.T., and K.W.W. acknowledge the support from the National Science Foundation (Award No. CMMI-1634545) and the University of Michigan Collegiate Professorship. S.L. acknowledges the support from the National Science Foundation (Awards No. CMMI-1633952, No. CMMI-1751449 CAREER, and No. CMMI-1760943) and Clemson University (startup funding and Dean's Faculty Fellow Award).

\section{APPENDIX A: CONSTRUCTIONS OF 2D BRAVAIS LATTICES WITH DEGREE-4 VERTEX ORIGAMIS}

There are five types of 2D Bravais lattices: oblique, rectangular, centered rectangular, square, and hexagonal lattices. For simplicity, they are hereafter referred to as $\mathrm{O}, \mathrm{R}, \mathrm{CR}, \mathrm{S}$, and $\mathrm{H}$, respectively. In this Appendix section, we show how they can be constructed based on degree-4 vertex (4-vertex) origamis by providing the detailed correlations between origami geometries and lattice configurations. Note that there are multiple origami designs and multiple characteristic entity assignments that can be used to create one specific type of 2D Bravais lattice. Thus, the constructions given here serve as examples to elucidate the underlying geometric correlations.

To obtain an oblique lattice, a single-collinear (SC) origami pattern is used. Figure 4(a) shows the geometry of an SC origami unit at both the flat and a partially folded state. An (a)

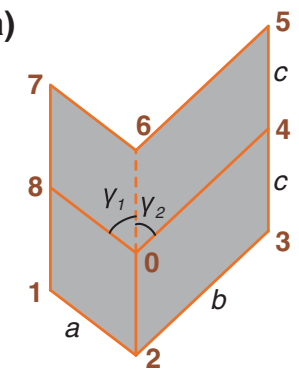

SC origami unit, crease pattern

(b)

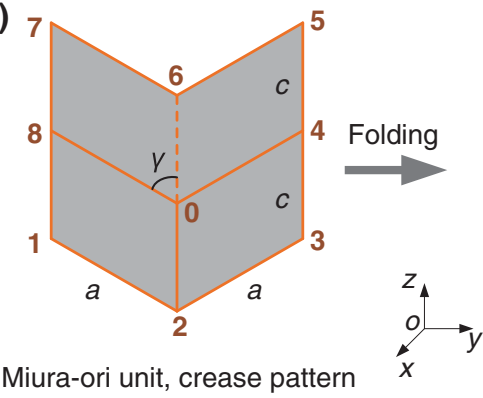

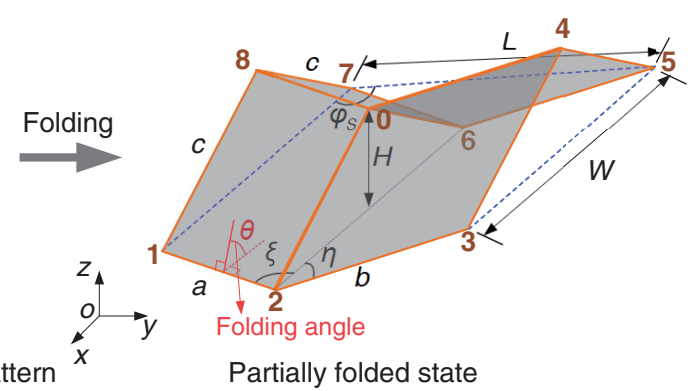

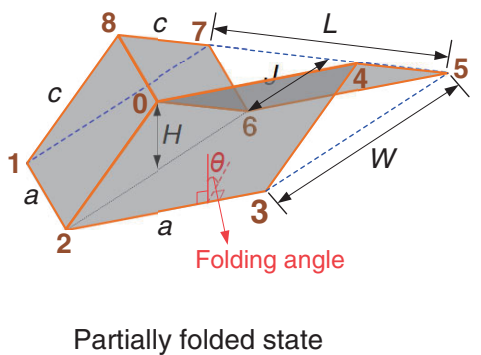

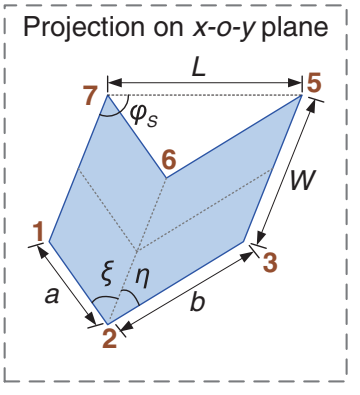

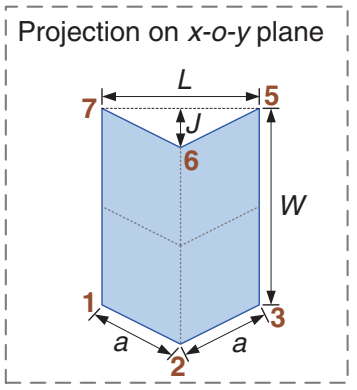

FIG. 4. Geometries of 2D origami units. (a) An SC origami unit, and (b) a Miura-ori unit. For each case, the crease pattern (left), the origami unit at a partially folded state (middle), and its projection onto the reference $x$ - $o-y$ plane (right) are given. In the crease patterns, "mountain" and "valley" creases are represented by solid and dashed lines, respectively. 


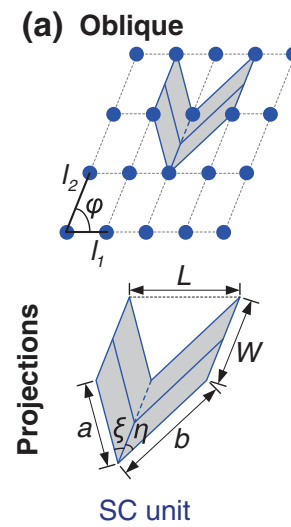

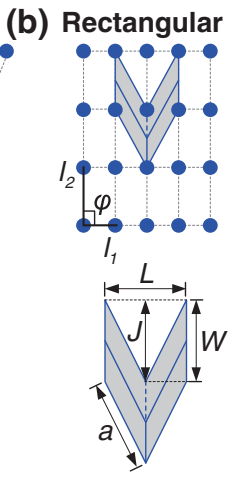

Miura-ori unit (c) Centered rectangular

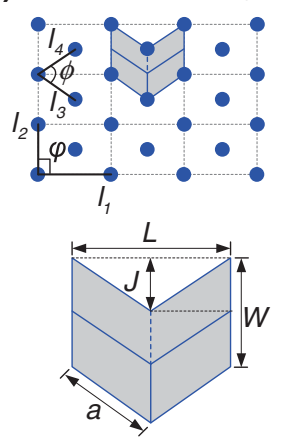

Miura-ori unit

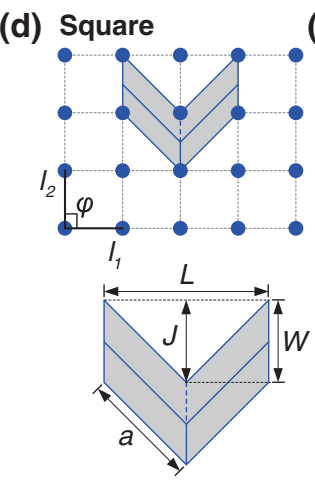

Miura-ori unit (e) Hexagonal

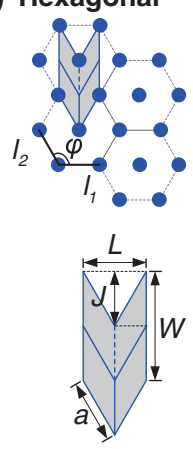

Miura-ori unit

FIG. 5. The five types of 2D Bravais lattices and the corresponding polygons projected from a partially folded origami unit. (a) The oblique lattice, (b) the rectangular lattice, (c) the centered rectangular lattice, (d) the square lattice, and (e) the hexagonal lattice.

$\mathrm{SC}$ unit can be characterized by three crease lengths $(a, b$, and $c$ ) and two sector angles $\left(\gamma_{1}\right.$ and $\left.\gamma_{2}\right)$ [left, Fig. 4(a)]. Folding of the SC unit can be described by the folding angle $\theta\left(\theta \in\left[0,90^{\circ}\right]\right)$, which is defined as the dihedral angle between the facet 1-2-0-8 (or facet 0-6-7-8) and the reference $x$-o-y plane. Vertices $1,2,3,5,6$, and 7 are all coplanar in the $x-o-y$ plane. The outer dimensions of the SC unit are given by [27]

$$
\begin{aligned}
\xi & =\arccos \frac{\cos \gamma_{1}}{\sqrt{1-\sin ^{2} \gamma_{1} \sin ^{2} \theta}}, \\
\eta & =\arccos \frac{\cos \gamma_{2}}{\sqrt{1-\sin ^{2} \gamma_{1} \sin ^{2} \theta}}, \\
\varphi_{S} & =\xi+\arccos \frac{a^{2}+L^{2}-b^{2}}{2 a L} \\
L & =\sqrt{a^{2}+b^{2}-2 a b \cos (\xi+\eta)}, \\
W & =2 c \sqrt{1-\sin \gamma^{2} \sin ^{2} \theta}, \\
H & =c \sin \gamma_{1} \sin \theta .
\end{aligned}
$$

where $H, W$, and $L$ are the height, width, and length of a folded SC unit, respectively [middle, Fig. 4(a)]. By projecting the folded SC unit onto the reference $x-o-y$ plane, we obtain a polygon, whose shape is described by angles $\xi, \eta$, and $\varphi_{S}$ [middle and right, Fig. 4(a)].
The other four types of 2D Bravais lattices can be constructed by using Miura-ori patterns. Figure 4(b) shows the geometry of a Miura-ori unit. A Miura-ori unit can be characterized by two crease lengths ( $a$ and $c$ ) and one sector angle $(\gamma)$ [left, Fig. 4(b)]. Folding of the Miura-ori unit can also be described by the folding angle $\theta\left(\theta \in\left[0,90^{\circ}\right]\right)$, defined as the dihedral angle between one of its facets and the reference $x-o-y$ plane. Vertices $1,2,3,5,6$, and 7 are coplanar in the $x-o-y$ plane. The outer dimensions of the Miura-ori unit are given by [22]

$$
\begin{aligned}
& H=c \sin \gamma \sin \theta, \quad W=2 c \sqrt{1-\sin ^{2} \gamma \sin ^{2} \theta}, \\
& L=2 a \frac{\cos \theta \sin \gamma}{\sqrt{1-\sin ^{2} \gamma \sin ^{2} \theta}}, \quad J=\frac{a}{\sqrt{1+\tan ^{2} \gamma \cos ^{2} \theta}},
\end{aligned}
$$

where $H, W, L$ are the height, width, and length of a folded Miura-ori unit, respectively [middle, Fig. 4(b)]. Projecting the folded unit onto the $x$-o- $y$ plane, a polygon is also obtained.

By treating the coplanar vertices $1,2,3,5,6$, and 7 as

\begin{tabular}{|c|c|c|c|c|}
\hline $\begin{array}{l}\text { 2D Bravais } \\
\text { lattices }\end{array}$ & $\begin{array}{l}\text { Point groups } \\
\text { (symmetries) }\end{array}$ & $\begin{array}{l}\text { Axial distances } \\
\text { and axial angles }\end{array}$ & $\begin{array}{l}\text { Origami } \\
\text { patterns }\end{array}$ & $\begin{array}{l}\text { Correlations between origami } \\
\text { geometries and lattice geometries }\end{array}$ \\
\hline Oblique $(\mathrm{O})$ & $C_{2}$ & $l_{1} \neq l_{2}, \quad \varphi \neq 90^{\circ}$ & $\mathrm{SC}$ & $\begin{array}{c}a=\sqrt{l_{1}^{2}+l_{2}^{2}-2 l_{1} l_{2} \cos \varphi}, \\
b=\sqrt{l_{1}^{2}+l_{2}^{2}+2 l_{1} l_{2} \cos \varphi}, \\
W=l_{2}, \quad L=2 l_{1}, \\
\xi=\arcsin \left(l_{1} \sin \varphi / a\right), \\
\eta=\arcsin \left(l_{1} \sin \varphi / b\right)\end{array}$ \\
\hline $\begin{array}{l}\text { Rectangular }(\mathrm{R}) \\
\text { Centered rectangular }(\mathrm{CR})\end{array}$ & $D_{2}$ & $\begin{array}{l}l_{1} \neq l_{2}, \quad \varphi=90^{\circ} \\
l_{1} \neq l_{2}, \quad \varphi=90^{\circ} \\
\left(l_{3} \neq l_{4}, \quad \phi \neq 90^{\circ}\right)\end{array}$ & Miura-ori & $\begin{array}{c}a=\sqrt{{l_{1}^{2}+l_{2}^{2}}^{2}}, \quad J=W=l_{2}, \quad L=2 l_{1} \\
a=l_{3}, \quad W=2 l_{3} \sin (\phi / 2), \\
J=W / 2, \quad L=2 l_{3} \cos (\phi / 2)\end{array}$ \\
\hline Square (S) & $D_{4}$ & $l_{1}=l_{2}, \quad \varphi=90^{\circ}$ & & $a=\sqrt{2} l_{1}, \quad J=W=l_{1}, \quad L=2 l_{1}$ \\
\hline Hexagonal $(\mathrm{H})$ & $D_{6}$ & $l_{1}=l_{2}, \quad \varphi=120^{\circ}$ & & $a=l_{1}, \quad W=\sqrt{3} l_{1}, \quad J=W / 2, \quad L=l_{1}$ \\
\hline
\end{tabular}
lattice points and placing inclusions on them, we can construct $2 \mathrm{D}$ origami lattices of vertex inclusions. Figure 5 shows the polygons that are projected from a partially folded origami unit and the corresponding 2D Bravais lattices. For each 2D Bravais lattice, the detailed correlations between the origami geometries and lattice geometries are given in Table I.

TABLE I. Geometry correlations for constructing 2D Bravais lattices based on 4-vertex origamis. 
(a)

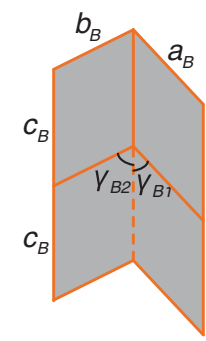

SC unit B

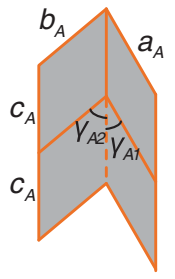

SC unit A

(b)
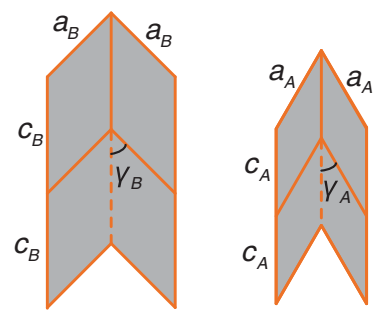

Miura-ori unit B Miura-ori unit A
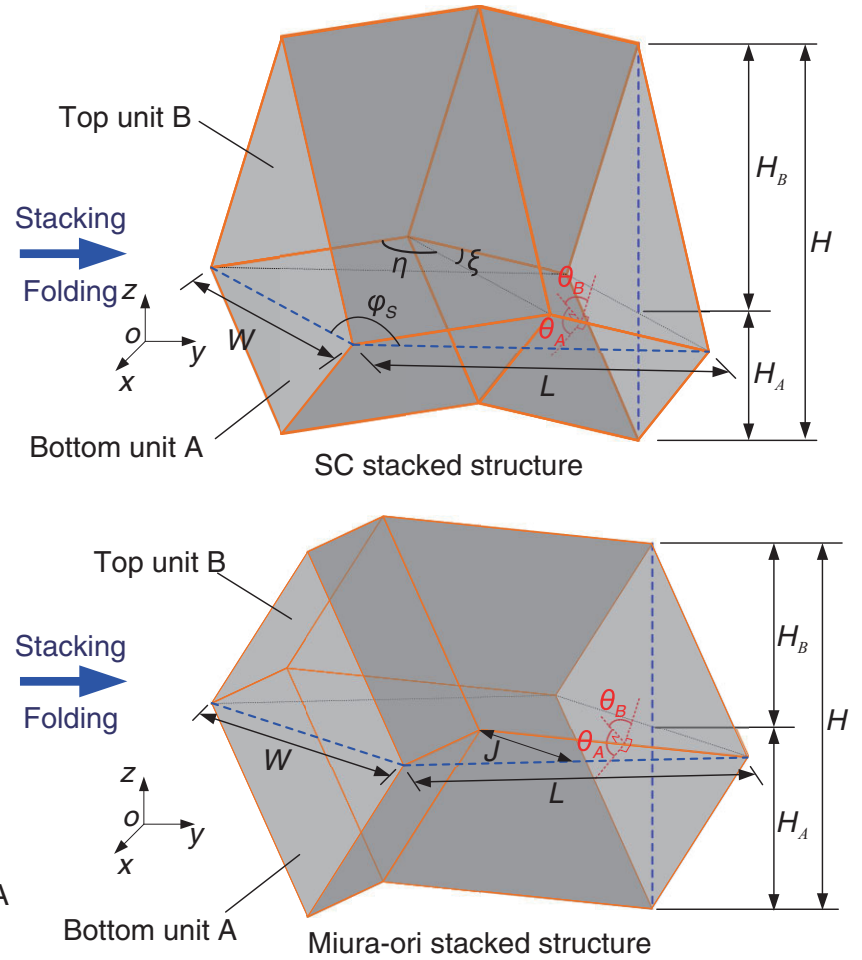

(c)

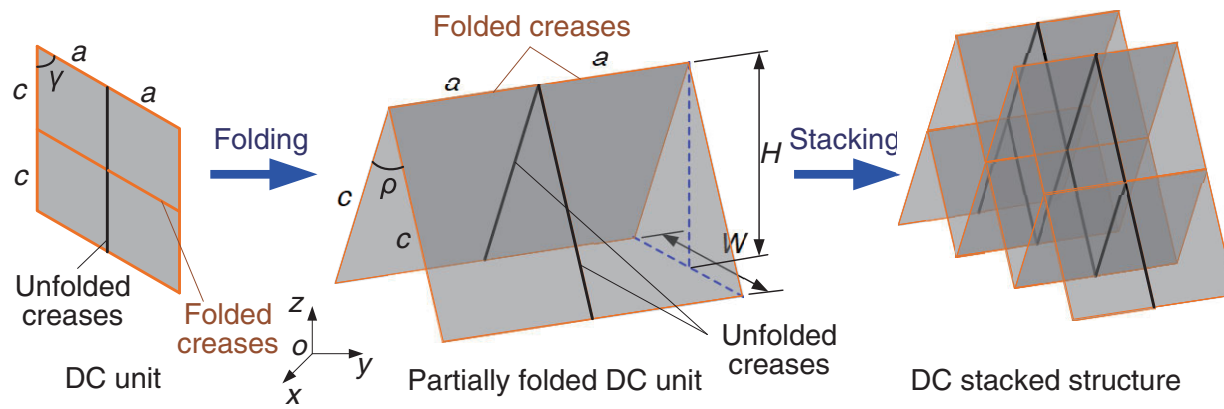

FIG. 6. Geometries of 3D origami structures. (a) A stacked SC structure (bulged out), (b) a stacked Miura-ori structure (bulged out), and (c) a partially folded DC unit and the corresponding DC stacked structure. For each case, the constituent units' crease patterns (left) and the stacked origami structure at a partially folded state (right) are given. In the crease patterns, the mountain and valley creases are denoted by solid and dashed lines, respectively. In the DC unit, the folded and unfolded creases are highlighted.

\section{APPENDIX B: CONSTRUCTIONS OF 3D BRAVAIS LATTICES WITH DEGREE-4 VERTEX ORIGAMIS}

Three-dimensional Bravais lattices can be categorized into seven systems. In each system, the lattice points in a unit cell can follow four different centering types. Specifically, the seven systems are triclinic, monoclinic, orthorhombic, tetragonal, rhombohedral, hexagonal, and cubic [42]. The four centering types are primitive, base-centered, body-centered, and face-centered. However, some combinations of lattice systems and centering types create the same lattice. After considering such redundancy, there are 14 unique types of 3D Bravais lattices.

We use three types of origami structures to construct 3D Bravais lattices. They are a stacked SC structure in the bulged-out configuration, a stacked Miura-ori structure in the bulged-out configuration, and a stacked double-collinear (DC) structure. In what follows, we formulate the geometries of the three stacked origami structures, and then detail the cor- relations between the origami geometries and the 3D lattice configurations.

The stacked SC structure consists of two SC units [Fig. 6(a)]. Each SC unit is characterized by crease lengths $a_{x}$, $b_{x}$, and $c_{x}$, and sector angles $\gamma_{x 1}$ and $\gamma_{x 2}$. Here the subscript $x$ takes the values of $A$ or $B$ and represents the bottom unit $A$ or the top unit $B$, respectively. Without loss of generality, we assume $\gamma_{x 1}<\gamma_{x 2}$. The following geometry constraints have to be satisfied to ensure the kinematic compatibility between the two SC units during the whole rigid-folding process [27]:

$$
a_{A}=a_{B}=a, \quad b_{A}=b_{B}=b, \quad \frac{\cos \gamma_{A 1}}{\cos \gamma_{B 1}}=\frac{\cos \gamma_{A 2}}{\cos \gamma_{B 2}}=\frac{c_{B}}{c_{A}} .
$$

Folding of the stacked SC structure is still a single-degreeof-freedom mechanism. It can be described by a folding angle $\theta_{A}$ (or $\theta_{B}$ ), which is defined as the dihedral angle between a facet of the bottom (or top) unit and the reference $x-o-y$ plane 
[Fig. 6(a)]. $\theta_{A}$ and $\theta_{B}$ are not independent of each other but satisfy the following relationship:

$$
\frac{\cos \theta_{A}}{\cos \theta_{B}}=\frac{\tan \gamma_{B 1}}{\tan \gamma_{A 1}} .
$$

In the bulged-out configuration $-90^{\circ} \leqslant \theta_{A} \leqslant 0$, and in the nested-in configuration $0<\theta_{A} \leqslant 90^{\circ}$. The outer dimensions of the SC stacked structure in the bulged-out configuration are

$$
\begin{aligned}
H_{A} & =c_{A} \sin \gamma_{A 1} \sin \left|\theta_{A}\right|, \quad H_{B}=c_{B} \sin \gamma_{B 1} \sin \theta_{B}, \\
H & =H_{A}+H_{B}, \quad \xi=\arccos \frac{\cos \gamma_{A 1}}{\sqrt{1-\sin ^{2} \gamma_{A 1} \sin ^{2} \theta_{A}}}, \\
\eta & =\arccos \frac{\cos \gamma_{A 2}}{\sqrt{1-\sin ^{2} \gamma_{A 1} \sin ^{2} \theta_{A}}}, \\
W & =2 c_{A} \sqrt{1-\sin ^{2} \gamma_{A 1} \sin ^{2} \theta_{A}}, \\
L & =\sqrt{a_{A}^{2}+b_{A}^{2}-2 a_{A} b_{A} \cos (\xi+\eta)}, \\
\varphi_{S} & =\xi+\arccos \frac{a_{A}^{2}+L^{2}-b_{A}^{2}}{2 a_{A} L} .
\end{aligned}
$$

The stacked SC structure is used to construct the triclinic and the monoclinic lattices. To characterize the triclinic lattice, two additional angles $\varphi_{1}$ and $\varphi_{2}$ [Fig. 7(a)] are defined:

$$
\begin{aligned}
& \varphi_{1}=\arccos \left(-\frac{W \cos \varphi_{S}}{2 a_{A}}\right), \\
& \varphi_{2}=\pi-\arcsin \left[\frac{\sqrt{c_{A}^{2}-(W / 2)^{2}}}{c_{A}}\right] .
\end{aligned}
$$

The stacked Miura-ori structure consists of two Miura-ori units [Fig. 6(b)]. Each Miura-ori unit is characterized by two crease lengths $a_{x}$ and $b_{x}$, and one sector angle $\gamma_{x}$, where the subscript $x$ also takes the values of $A$ or $B$, denoting the bottom unit $A$ or the top unit $B$, respectively. The following geometry constraints have to be satisfied to ensure the kinematic compatibility between the two units during rigid folding [22]:

$$
a_{A}=a_{B}=a, \quad \frac{\cos \gamma_{A}}{\cos \gamma_{B}}=\frac{c_{B}}{c_{A}} .
$$

Similarly, folding of the stacked Miura-ori structure is a single-degree-of-freedom mechanism. It can be described by a folding angle $\theta_{A}$ (or $\theta_{B}$ ), which is defined as the dihedral angle between a facet of the bottom (or top) unit and the reference $x$-o-y plane [Fig. 6(b)]. $\theta_{A}$ and $\theta_{B}$ are not independent of each other but satisfy the following relationship:

$$
\frac{\cos \theta_{A}}{\cos \theta_{B}}=\frac{\tan \gamma_{B}}{\tan \gamma_{A}} .
$$

In the bulged-out configuration $-90^{\circ} \leqslant \theta_{A} \leqslant 0$, and in the nested-in configuration $0<\theta_{A} \leqslant 90^{\circ}$. The outer dimensions of the Miura-ori stacked structure in the bulged-out configuration are

$$
\begin{aligned}
H_{A} & =c_{A} \sin \gamma_{A} \sin \left|\theta_{A}\right|, \quad H_{B}=c_{B} \sin \gamma_{B} \sin \theta_{B}, \\
H & =H_{A}+H_{B}, \quad L=2 a_{A} \frac{\cos \theta_{A} \sin \gamma_{A}}{\sqrt{1-\sin ^{2} \gamma_{A} \sin ^{2} \theta_{A}}}, \\
J & =\frac{a_{A}}{\sqrt{1+\tan ^{2} \gamma_{A} \cos ^{2} \theta_{A}}}, \quad W=2 c_{A} \sqrt{1-\sin ^{2} \gamma_{A} \sin ^{2} \theta_{A}} .
\end{aligned}
$$

The stacked Miura-ori structure is used to construct the orthorhombic, the tetragonal, the hexagonal, and the cubic lattices.

The stacked DC structure is obtained by stacking two identical DC units [Fig. 6(c)]. Each DC unit has two pairs of creases that are collinear, and it can be characterized by two crease lengths $a, c$ and one sector angle $\gamma$. Folding of a DC unit can be described by the dihedral angle $\rho$ between its facets. Hence, the outer dimensions of a folded DC unit are

$$
H=c \cos (\rho / 2), \quad W=2 c \sin (\rho / 2) .
$$

The stacked DC structure is used to construct a specific rhombohedral lattice with axial angles equaling $60^{\circ}$. To obtain this lattice, two collinear creases have to remain unfolded.

Based on these three types of stacked origami structures, the 14 types of 3D Bravais lattices can be constructed according to Fig. 7. For each 3D Bravais lattice, a unit cell is shown on the corresponding origami structure. The detailed correlations between the origami geometries and the lattice geometries are listed in Table II.

\section{APPENDIX C: ACHIEVING DIFFUSIONLESS PHASE TRANSFORMATIONS THROUGH ORIGAMI FOLDING}

Diffusionless phase transformations can be distinguished between transformations dominated by lattice-distortive strains and those where shuffles play a significant role. Lattice-distortive strains can transform the lattice from one Bravais type to another, and the shuffle refers to small movements of a lattice point within the lattice unit cell. In this section, we thoroughly examine the examples given in Fig. 2 (detailed parameters of these examples are provided in Table III). We show that based on different origami patterns, all types of 2D and 3D diffusionless transformations can be achieved by rigid folding. For each type of transformation, a lattice transformation matrix is formulated, which is further correlated to the kinematic properties of 2D and 3D origami structues. Such correlations are summarized in Table S4 in the Supplemental Material [31].

\section{2D Dilation}

Two-dimensional dilation is observed in a lattice of vertex inclusions based on a Miura-ori, which is an example of 2D Bravais lattice shown in Figs. 2(a) and 8(a). Folding of a Miura-ori unit can be described by the dihedral folding angle $\theta\left(\theta \in\left[0,90^{\circ}\right]\right)$ between its facet and the reference $x$ - $o-y$ plane [Fig. 4(b)]. Hence, the lattice vectors corresponding to two different configurations (with folding angles $\theta$ and $\theta^{\prime}$ ) can be formulated as

$\mathbf{l}_{1}^{\theta}=\left(\begin{array}{c}\left.L\right|_{\theta} \\ 0\end{array}\right), \quad \mathbf{l}_{2}^{\theta}=\left(\begin{array}{c}0 \\ \left.W\right|_{\theta}\end{array}\right) ; \quad \mathbf{l}_{1}^{\theta^{\prime}}=\left(\begin{array}{c}\left.L\right|_{\theta^{\prime}} \\ 0\end{array}\right), \quad \mathbf{l}_{2}^{\theta^{\prime}}=\left(\begin{array}{c}0 \\ \left.W\right|_{\theta^{\prime}}\end{array}\right)$.

Here, the length $L$ and the width $W$ of the Miura-ori unit are defined in Eq. (A2). Since there is no diffusion involved, we can formulate a transformation matrix $\mathbf{U}_{2 D-D}$ to quantitatively describe the lattice transformation due to folding from angle 
(a) Triclinic: primitive

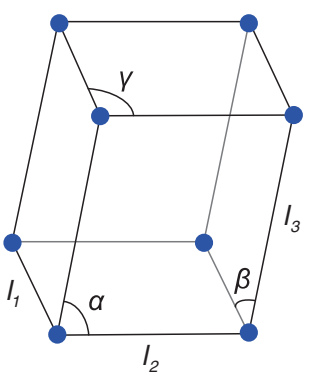

(b) (1) Monoclinic: primitive
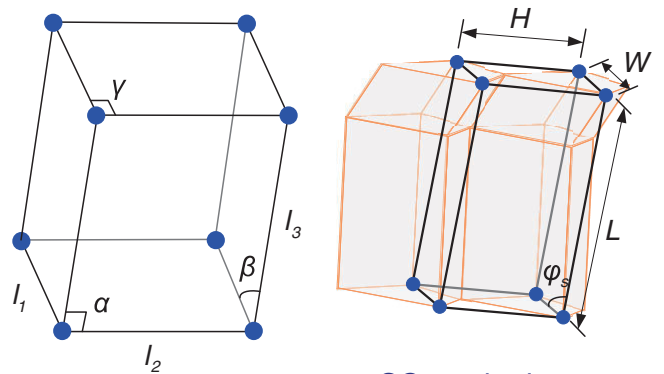

SC stacked structure

(c) (1) Orthorhombic: primitive
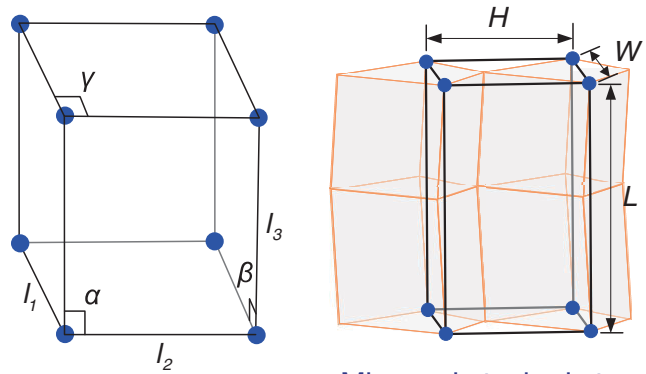

Miura-ori stacked structure

(3) Orthorhombic: body-centered

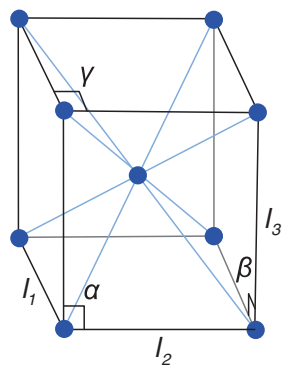

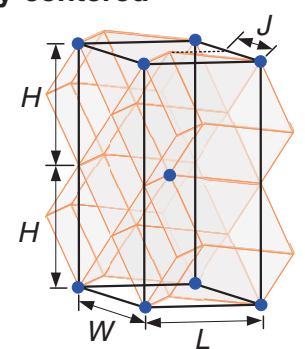

Miura-ori stacked structure
(2) Monoclinic: base-centered
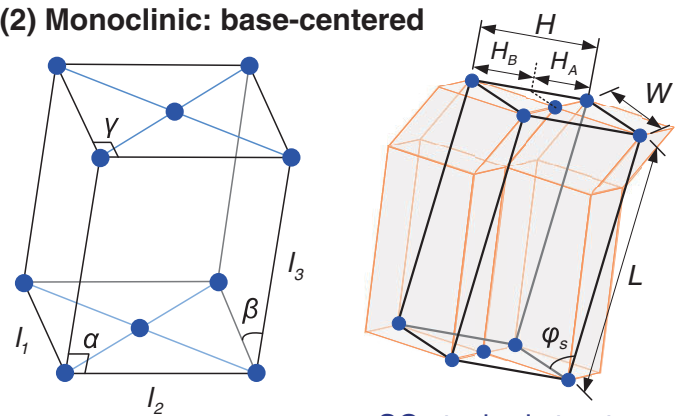

SC stacked structure

(2) Orthorhombic: base-centered
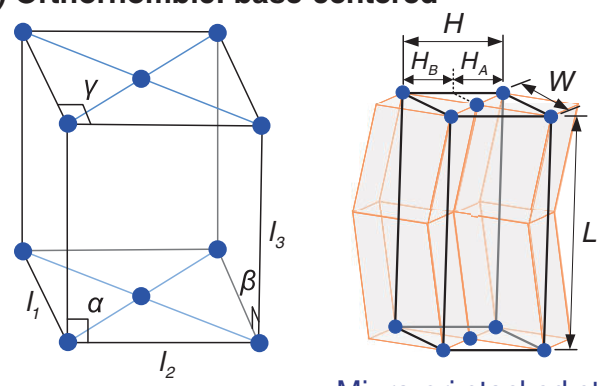

Miura-ori stacked structure

(4) Orthorhombic: face-centered

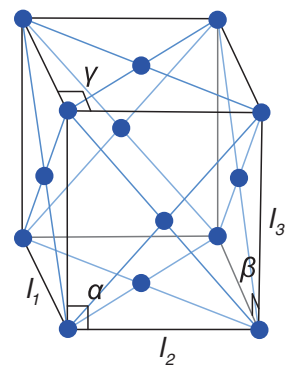

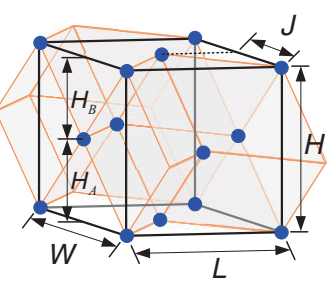

Miura-ori stacked structure

FIG. 7. The 14 types of 3D Bravais lattices and the layout of a lattice unit cell on the corresponding origami structure. (a) The triclinic lattice (primitive), (b) the monoclinic lattices (primitive and base-centered), and (c) the orthorhombic lattices (primitive, base-centered, body-centered, and face-centered), (d) the tetragonal lattices (primitive and base-centered), (e) the rhombohedral lattice (primitive), (f) the hexagonal lattice (primitive), and (g) the cubic lattices (primitive, base-centered, and face-centered).

$\theta$ to $\theta^{\prime}$ such that

$$
\left(\mathbf{l}_{1}^{\theta^{\prime}} \quad \mathbf{l}_{2}^{\theta^{\prime}}\right)^{T}=\mathbf{U}_{2 \mathrm{D}-\mathrm{D}}\left(\mathbf{l}_{1}^{\theta} \mathbf{l}_{2}^{\theta}\right)^{T} .
$$

$\mathbf{U}_{2 D-D}$ describes the lattice-distortive strains that transform the lattice (also called the Bain matrix), in which the subscript "2D" indicates that this transformation is two dimensional, and "D" means dilation. Substituting the expressions of $L$ and $W$ into Eqs. (C1) and (C2), the transformation matrix can be written as

$$
\begin{aligned}
\mathbf{U}_{2 \mathrm{D}-\mathrm{D}} & =\left(\begin{array}{cc}
s \cos \theta^{\prime} / \cos \theta & 0 \\
0 & 1 / s
\end{array}\right), \\
\text { where } s & =\frac{\sqrt{1-\sin ^{2} \gamma \sin ^{2} \theta}}{\sqrt{1-\sin ^{2} \gamma \sin ^{2} \theta^{\prime}}} .
\end{aligned}
$$


(d) (1) Tetragonal: primitive
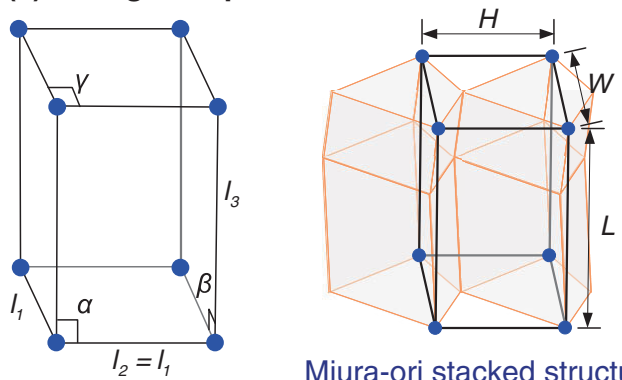

Miura-ori stacked structure

(e) Rhombohedral: primitive
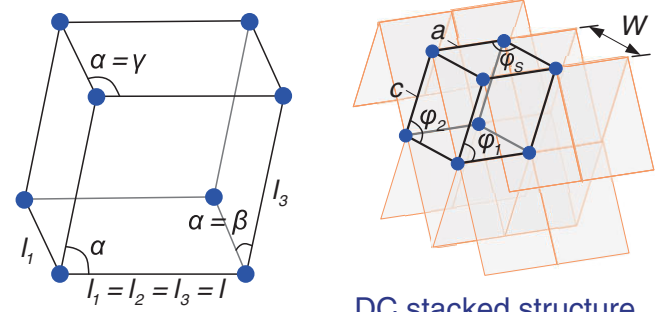

DC stacked structure

(g) (1) Cubic: primitive

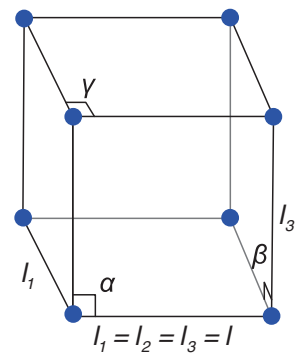

Miura-ori stacked structure
(2) Tetragonal: base-centered
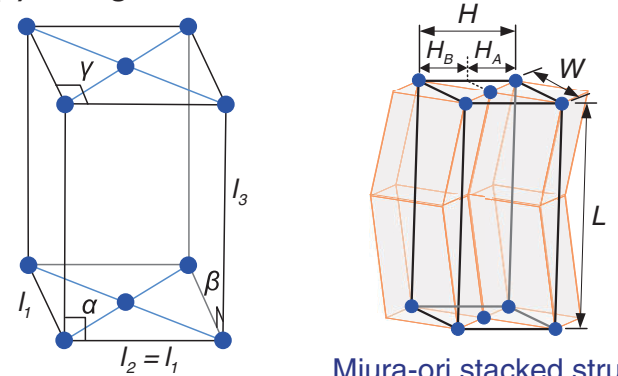

Miura-ori stacked structure

(f) Hexagonal: primitive
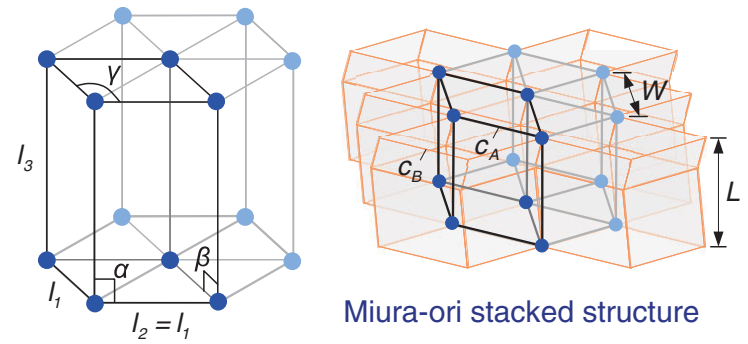

Miura-ori stacked structure

(2) Cubic: body-centered
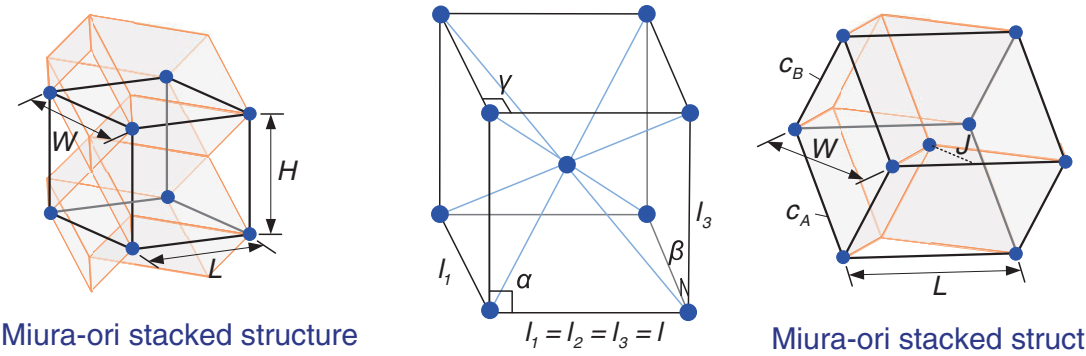

Miura-ori stacked structure

(3) Cubic: face-centered

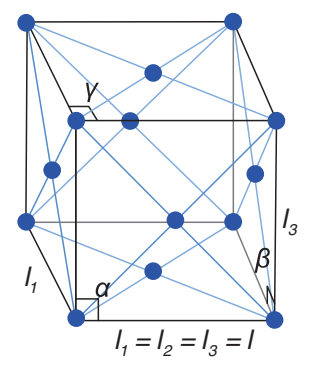

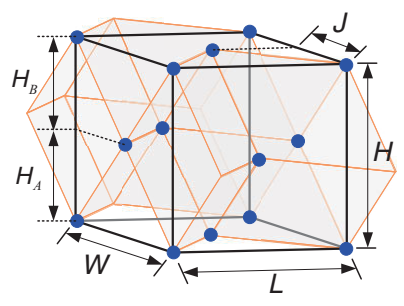

Miura-ori stacked structure

FIG. 7. (Continued.).

$\mathbf{U}_{2 \mathrm{D}-\mathrm{D}}$ is a diagonal matrix. When $\theta^{\prime}<\theta$, the two diagonal elements of $\mathbf{U}_{\text {2D-D }}$ are always larger than 1, suggesting that the lattice dilates along both lattice vectors.

Note that the Miura-ori unit exhibits a negative Poisson's ratio in the $L$ and $W$ directions during folding [22]:

$$
v_{W L}=-\frac{\varepsilon_{W}}{\varepsilon_{L}}=-\frac{\mathrm{d} W / W}{\mathrm{~d} L / L}=-\cos ^{2} \theta \tan ^{2} \gamma<0 .
$$

When the length $L$ increases (or decreases) due to folding, the width $W$ increases (or decrease) accordingly. Therefore, the negative Poisson's ratio is the kinematic origin of the 2D dilation.

\section{2D Contraction and extension}

Two-dimensional contraction and extension is observed when transforming an eggbox-pattern-based lattice of vertex inclusions, which is an example of 2D Bravais lattice shown in Figs. 2(b) and 8(b). The eggbox pattern [29] consists of four identical parallelogram facets characterized by crease lengths $a, b$ and a sector angle $\gamma$; its length $L$ and width $W$ are given by

$$
W=2 a \sin \alpha, \quad L=2 b \sin \beta,
$$

where $\alpha$ and $\beta$ are two angles between the boundary creases and a perpendicular line, used for describing the folding motion $(0 \leqslant \alpha, \beta \leqslant \gamma)$ [Fig. 8(b)]. They are not independent of each other but follow the relationship $\cos \alpha \cos \beta=\cos \gamma$. 
TABLE II. Geometry correlations for constructing 3D Bravais lattices based on 4-vertex origami structures.

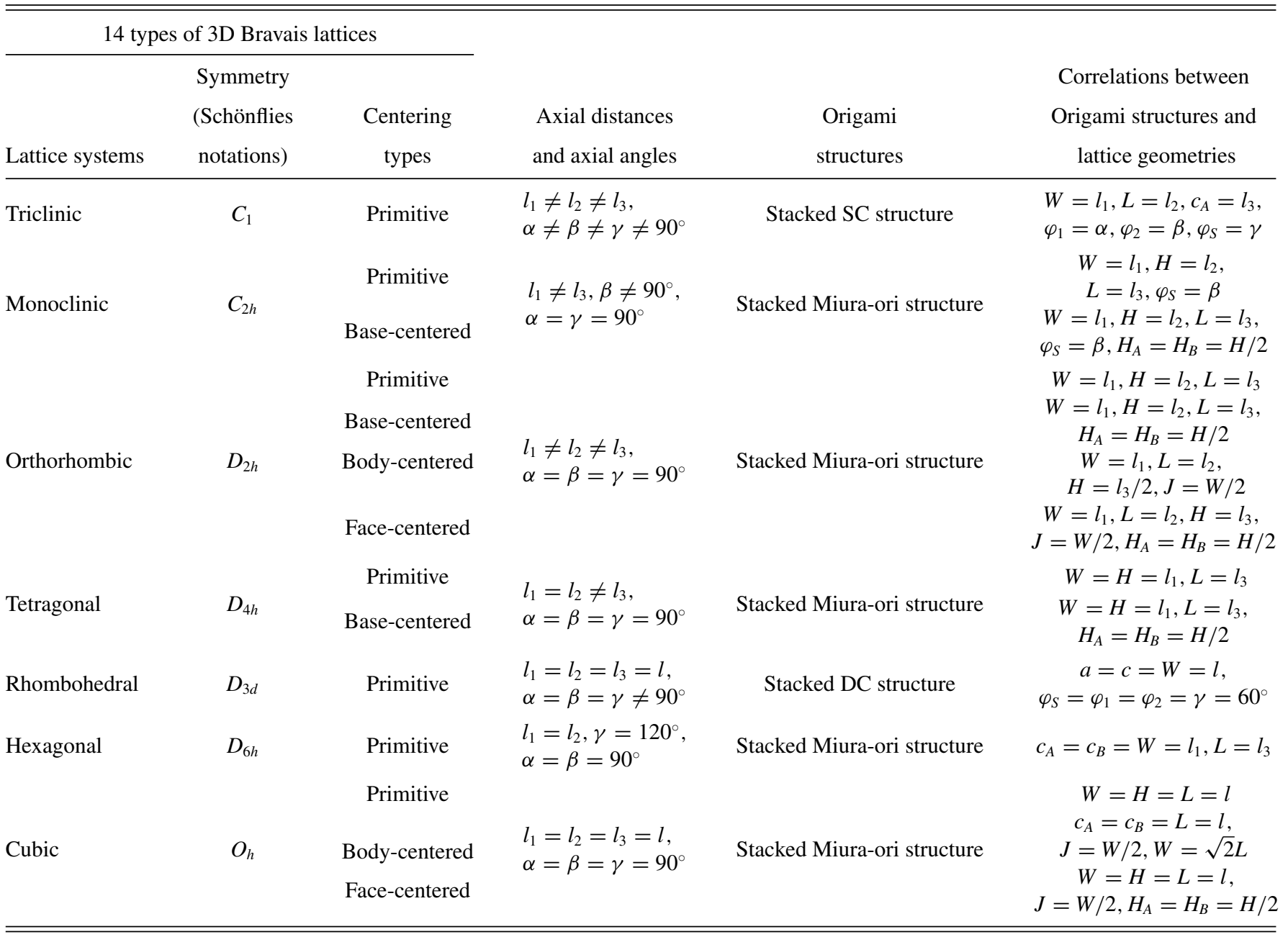

Hence, the lattice vectors corresponding to two different configurations (with angles $\alpha$ and $\alpha^{\prime}$ ) can be expressed as

$$
\begin{aligned}
\mathbf{l}_{1}^{\alpha}=\left(\begin{array}{c}
\left.L\right|_{\alpha} \\
0
\end{array}\right), \quad \mathbf{l}_{2}^{\alpha}=\left(\begin{array}{c}
0 \\
\left.W\right|_{\alpha}
\end{array}\right) ; \\
\mathbf{l}_{1}^{\alpha^{\prime}}=\left(\begin{array}{c}
\left.L\right|_{\alpha^{\prime}} \\
0
\end{array}\right), \quad \mathbf{l}_{2}^{\alpha^{\prime}}=\left(\begin{array}{c}
0 \\
\left.W\right|_{\alpha^{\prime}}
\end{array}\right) .
\end{aligned}
$$

Since there is no diffusion, the folding-induced lattice transformation from angle $\alpha$ to $\alpha^{\prime}$ can be described by a transformation matrix $\mathbf{U}_{2 \mathrm{D}-\mathrm{C} / \mathrm{E}}$ such that

$$
\left(\begin{array}{ll}
\mathbf{l}_{1}^{\alpha^{\prime}} & \mathbf{l}_{2}^{\alpha^{\prime}}
\end{array}\right)^{T}=\mathbf{U}_{2 \mathrm{D}-\mathrm{C} / \mathrm{E}}\left(\begin{array}{ll}
\mathbf{l}_{1}^{\alpha} & \mathbf{l}_{2}^{\alpha}
\end{array}\right)^{T} .
$$

Here $\mathbf{U}_{2 \mathrm{D}-\mathrm{C} / \mathrm{E}}$ describes the lattice-distortive strains that

\begin{tabular}{|c|c|c|c|c|c|}
\hline \multicolumn{2}{|c|}{ Origami patterns used in Fig. 2} & \multicolumn{2}{|c|}{ Folding angles } & \multicolumn{2}{|c|}{ Transformation types } \\
\hline Patterns & Parameters & Angles & $\begin{array}{c}\text { Values used } \\
\text { in Fig. } 2\end{array}$ & $2 \mathrm{D}$ or $3 \mathrm{D}$ & Types \\
\hline Miura-ori sheet & $a=c, \gamma=60^{\circ}$ & $\theta$ & $60^{\circ} \rightarrow 30^{\circ}$ & & Dilation \\
\hline Eggbox sheet & $a=b, \gamma=60^{\circ}$ & $\alpha$ & $50^{\circ} \rightarrow 45^{\circ}$ & $2 \mathrm{D}$ & Contraction and extension \\
\hline SC sheet & $a=b=c, \gamma_{1}=36^{\circ}, \gamma_{2}=72^{\circ}$ & $\theta$ & $15^{\circ} \rightarrow 65^{\circ}$ & & With shear \\
\hline Miura-ori sheet & $c / a=0.7, \gamma=60^{\circ}$ & $\theta$ & $39.2^{\circ} \rightarrow 50^{\circ}$ & & With shuffle \\
\hline Stacked GFF structure (bulged out) & $\begin{array}{l}a_{A}=c_{A}, \gamma_{B 1}=54^{\circ} \\
\gamma_{A 1}=36^{\circ}, \gamma_{A 2}=72^{\circ}\end{array}$ & $\rho_{A 1}$ & $10^{\circ} \rightarrow 40^{\circ}$ & & Dilation \\
\hline Stacked Miura-ori structure (bulged out) & $a_{A}=c_{A}, \gamma_{A}=60^{\circ}, \gamma_{B}=75^{\circ}$ & $\theta_{A}$ & $-20^{\circ} \rightarrow-60^{\circ}$ & $3 \mathrm{D}$ & Contraction and extension \\
\hline GFF sheet & $a=c, \gamma_{1}=36^{\circ}, \gamma_{2}=72^{\circ}$ & $\rho_{1}$ & $90^{\circ} \rightarrow 30^{\circ}$ & & With shear \\
\hline Stacked Miura-ori structure (nested in) & $a_{A}=c_{A}, \gamma_{A}=60^{\circ}, \gamma_{B}=75^{\circ}$ & $\theta_{A}$ & $20^{\circ} \rightarrow 60^{\circ}$ & & With shuffle \\
\hline
\end{tabular}
transform the lattice; the subscript "2D-C/E" stands for

TABLE III. Detailed geometries and folding angles of the origami patterns used in Fig. 2. 
(a) 2D dilation based on a Miura-ori sheet

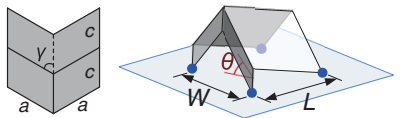

Crease pattern Folded Miura-ori unit

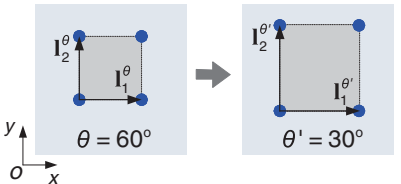

(c) 2D shear component

based on a SC sheet
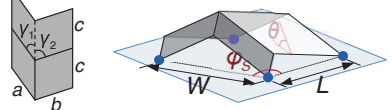

Crease pattern Folded SC unit

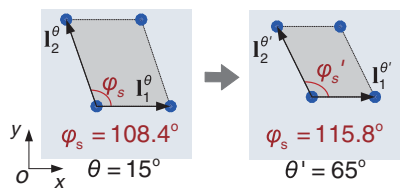

(b) 2D contraction and extension based on an eggbox sheet
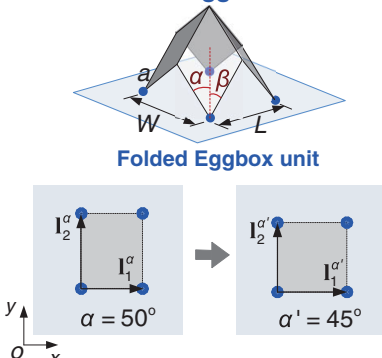

(d) 2D shuffle component

based on a Miura-ori sheet

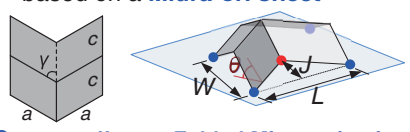

Crease pattern Folded Miura-ori unit

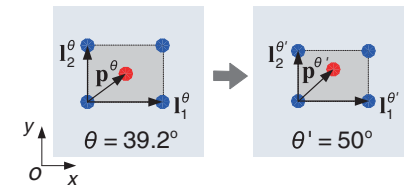

FIG. 8. Origami geometries and 2D diffusionless phase transformations. (a) $2 \mathrm{D}$ dilation achieved by transforming a Miura-oribased lattice of vertex inclusions; (b) 2D contraction and extension achieved by transforming an eggbox-pattern-based lattice of vertex inclusions; (c) 2D shear involved in transforming an SC-origamibased lattice of vertex inclusions; (d) 2D shuffle involved in transforming a Miura-ori-based lattice of vertex inclusions.

two-dimensional contraction and extension. Substituting the expressions of $L$ and $W$ [i.e., Eq. (C5)] into Eqs. (C6) and (C7), the transformation matrix yields

$$
\mathbf{U}_{2 \mathrm{D}-\mathrm{C} / \mathrm{E}}=\left(\begin{array}{cc}
\sin \beta^{\prime} / \sin \beta & 0 \\
0 & \sin \alpha^{\prime} / \sin \alpha
\end{array}\right) .
$$

$\mathbf{U}_{2 \mathrm{D}-\mathrm{C} / \mathrm{E}}$ is also a diagonal matrix. When $\alpha^{\prime}>\alpha$, $\sin \alpha^{\prime} / \sin \alpha>1$ but $0<\sin \beta^{\prime} / \sin \beta<1$, suggesting that the lattice contracts along one lattice vector but expands along the other vector, and vice versa.

Note that the eggbox unit exhibits a positive Poisson's ratio in the $L$ and $W$ directions during folding [29]:

$$
v_{W L}=-\frac{\varepsilon_{W}}{\varepsilon_{L}}=-\frac{\mathrm{d} W / W}{\mathrm{~d} L / L}=\frac{\cos ^{2} \alpha-\cos ^{2} \gamma}{\cos ^{2} \theta \tan ^{2} \gamma}>0 .
$$

When the length $L$ increases due to folding, the width $W$ decreases instead, and vice versa. Therefore, the positive Poisson's ratio is the kinematic origin of the 2D contraction and extension.

\section{2D Shear}

A two-dimensional shear component is observed when we transform a lattice of vertex inclusions based on a single collinear (SC) origami, which is an example of 2D Bravais lattice shown in Figs. 2(c) and 8(c). Folding of the SC unit can be described by the dihedral angle $\theta\left(\theta \in\left[0,90^{\circ}\right]\right)$ defined between the facet and the reference $x-o-y$ plane [Fig. 4(a)] [27], so that the lattice vectors corresponding to two different

configurations (with folding angles $\theta$ and $\theta^{\prime}$ ) yield

$$
\begin{aligned}
& \mathbf{l}_{1}^{\theta}=\left(\begin{array}{c}
\left.L\right|_{\theta} \\
0
\end{array}\right), \quad \mathbf{l}_{2}^{\theta}=\left[\begin{array}{c}
\left.\left(W \cos \varphi_{S}\right)\right|_{\theta} \\
\left.\left(W \sin \varphi_{S}\right)\right|_{\theta}
\end{array}\right] ; \\
& \mathbf{l}_{1}^{\theta^{\prime}}=\left(\begin{array}{c}
\left.L\right|_{\theta^{\prime}} \\
0
\end{array}\right), \quad \mathbf{l}_{2}^{\theta^{\prime}}=\left[\begin{array}{c}
\left.\left(W \cos \varphi_{S}\right)\right|_{\theta^{\prime}} \\
\left.\left(W \sin \varphi_{S}\right)\right|_{\theta^{\prime}}
\end{array}\right] .
\end{aligned}
$$

The length $L$, width $W$, and the angle $\varphi_{S}$ of the $\mathrm{SC}$ unit are defined in Eq. (A1). Since there is no diffusion, the folding-induced lattice transformation from angle $\theta$ to $\theta^{\prime}$ can be described by a transformation matrix $\mathbf{U}_{2 \mathrm{D}-\mathrm{S}}$ such that

$$
\left(\begin{array}{ll}
\mathbf{l}_{1}^{\theta^{\prime}} & \mathbf{l}_{2}^{\theta^{\prime}}
\end{array}\right)^{T}=\mathbf{U}_{2 \mathrm{D}-\mathrm{S}}\left(\begin{array}{ll}
\mathbf{l}_{1}^{\theta} & \mathbf{l}_{2}^{\theta}
\end{array}\right)^{T} .
$$

Here $\mathbf{U}_{2 D-S}$ describes the lattice-distortive strains that transform the lattice; the subscript " $2 \mathrm{D}-\mathrm{S}$ " means two-dimensional shear. $\mathbf{U}_{2 D-S}$ can be formulated as

$$
\mathbf{U}_{2 \mathrm{D}-\mathrm{S}}=\left(\begin{array}{cc}
\left.L\right|_{\theta^{\prime}} /\left.L\right|_{\theta} & 0 \\
\frac{\left.W\right|_{\theta^{\prime}}\left(\left.\cos \varphi_{S}\right|_{\theta^{\prime}}-\left.\sin \varphi_{S}\right|_{\theta^{\prime}} /\left.\tan \varphi_{S}\right|_{\theta}\right)}{\left.L\right|_{\theta}} & \frac{\left.\left(W \sin \varphi_{S}\right)\right|_{\theta^{\prime}}}{\left.\left(W \sin \varphi_{S}\right)\right|_{\theta}}
\end{array}\right) .
$$

$\mathbf{U}_{2 D-S}$ is not a diagonal matrix. In addition to the nontrivial diagonal elements that describes the contraction and (or) extension along the lattice vectors, there is also a nonzero off-diagonal element describing the distortion induced by shearing. By substituting the expressions of $L, W$, and $\varphi_{S}$ [i.e., Eq. (A1)] into Eq. (C12), correlations between the transformation matrix $\mathbf{U}_{2 D-S}$ and the rigid-folding kinematics can be established.

Note that the SC origami is characterized by its ability in achieving in-plane shearing deformation during folding, which manifests as the changes of the angle $\varphi_{S}$. For example, by folding the $\mathrm{SC}$ unit $\left(a=b=c, \gamma_{1}=36^{\circ}, \gamma_{2}=72^{\circ}\right)$ from $\theta=15^{\circ}$ to $\theta=65^{\circ}$, the angle $\varphi_{S}$ changes from $108.4^{\circ}$ to $115.8^{\circ}$. As a result, the in-plane shearing deformation mechanism is the root cause of the $2 \mathrm{D}$ shear component in lattice transformations.

\section{2D Shuffle}

Two-dimensional shuffle plays a significant role in transforming a particular type of Miura-ori-based lattice of vertex inclusions shown in Figs. 2(d) and 8(d). Generally, this lattice as a whole is not a Bravais type due to the additional lattice point within the unit cell. As a result, the two lattice vectors used in the previous three cases are no longer sufficient to describe this lattice, because the lattice point inside the unit cell cannot be obtained by translating the two vectors. This kind of lattice is essentially a collection of two congruent Bravais lattices that are shifted from one another. Mathematically, such multilattice [43] can be characterized by a group of lattice vectors $\mathbf{l}_{\mathbf{i}}$ and a shift vector $\mathbf{p}$. That is, $\mathbf{l}_{\mathbf{i}}$ describes the constituent Bravais lattice, and $\mathbf{p}$ describes the offset between the two congruent lattices. Here, folding of the Miura-ori unit is still described by the dihedral angle $\theta\left(\theta \in\left[0,90^{\circ}\right]\right)$ between its facet and the reference $x-o-y$ plane [Fig. 4(a)] [27], so that the vectors corresponding to two different configurations (with folding angles $\theta$ and $\theta^{\prime}$ ) can be expressed as

$$
\begin{aligned}
& \mathbf{l}_{1}^{\theta}=\left(\begin{array}{c}
\left.L\right|_{\theta} \\
0
\end{array}\right), \quad \mathbf{l}_{2}^{\theta}=\left(\begin{array}{c}
0 \\
\left.W\right|_{\theta}
\end{array}\right), \quad \mathbf{p}^{\theta}=\left(\begin{array}{c}
\left.L\right|_{\theta} / 2 \\
\left.J\right|_{\theta}
\end{array}\right) ; \\
& \mathbf{l}_{1}^{\theta^{\prime}}=\left(\begin{array}{c}
\left.L\right|_{\theta^{\prime}} \\
0
\end{array}\right), \quad \mathbf{l}_{2}^{\theta^{\prime}}=\left(\begin{array}{c}
0 \\
\left.W\right|_{\theta^{\prime}}
\end{array}\right), \quad \mathbf{p}^{\theta^{\prime}}=\left(\begin{array}{c}
\left.L\right|_{\theta^{\prime}} / 2 \\
\left.J\right|_{\theta^{\prime}}
\end{array}\right) .
\end{aligned}
$$


The dimensions $L, W$, and $J$ of the Miura-ori unit are defined in Eq. (A2). Since there is no diffusion, we can then describe the folding-induced lattice transformation from angle $\theta$ to $\theta^{\prime}$ as a combination of deformation and shift. Therefore, we can formulate the transformation matrix $\mathbf{T}_{2 \mathrm{D}-\mathrm{SH}}$ such that

$$
\left(\begin{array}{lll}
\mathbf{l}_{1}^{\theta^{\prime}} & \mathbf{l}_{2}^{\theta^{\prime}} & \mathbf{p}^{\theta^{\prime}}
\end{array}\right)^{T}=\mathbf{T}_{2 \mathrm{D}-\mathrm{SH}}\left(\mathbf{l}_{1}^{\theta} \mathbf{l}_{2}^{\theta} \mathbf{p}^{\theta}\right)^{T} .
$$

Here the subscript "2D-SH" means two-dimensional shuffle, and $\mathbf{T}_{2 \mathrm{D}-\mathrm{SH}}$ can be written as

$$
\mathbf{T}_{2 \mathrm{D}-\mathrm{SH}}=\left(\begin{array}{ccc}
\mathbf{U}_{2 \mathrm{D}-\mathrm{D}} & 0 \\
\mu_{1} & \mu_{2} & \lambda
\end{array}\right) .
$$

$\mathbf{T}_{2 \mathrm{D}-\mathrm{SH}}$ is not a diagonal matrix, and it reflects how the latticedistortive strain and the shuffle component coexist in the transformation. $\mathbf{U}_{2 \mathrm{D}-\mathrm{D}}$ is the submatrix corresponding to $2 \mathrm{D}$ dilation, which describes the lattice-distortive strains of the constituent 2D Bravias lattice. It can be replaced by other transformation matrices (i.e., $\mathbf{U}_{2 D-D}, \mathbf{U}_{2 D-C / E}$, or $\mathbf{U}_{2 D-S}$ ) if different origami structures are employed. Other elements in the third row of $\mathbf{T}_{2 \mathrm{D}-\mathrm{SH}}$ quantify the $2 \mathrm{D}$ shuffle component, which satisfy the following relationship:

$$
\left(\mathbf{p}^{\theta^{\prime}}\right)^{T}=\left(\begin{array}{lll}
\mu_{1} & \mu_{2} & \lambda
\end{array}\right)\left(\begin{array}{lll}
\mathbf{l}_{1}^{\theta} & \mathbf{l}_{2}^{\theta} & \mathbf{p}^{\theta}
\end{array}\right)^{T} .
$$

Substituting the expressions of $L, W$, and $J$ [i.e., Eq. (A2)] into Eqs. (C13)-(C15), correlations between the matrix $\mathbf{T}_{2 \mathrm{D}-\mathrm{SH}}$ and the rigid-folding kinematics can be established.

Note that changes in the relative positions among vertices (or other characteristic entities) are ubiquitous in origami folding, and it is the underlying mechanism that induces the shuffle in lattice transformation. The shuffle component can be significant, and it can even move a lattice point from one unit cell to another, triggering a break and reconstruction of the nearest-neighbor relationship [e.g., Fig. 3(c) in the main text].

\section{3D Dilation}

Three-dimensional dilation is observed when transforming a lattice of vertex inclusions based on a stacked generic-flatfoldable (GFF) structure in the bulged-out configurations [27] [a kind of 3D Bravias lattice shown in Figs. 2(e) and 9(c)]. The stacked GFF structure consists of two GFF units $A$ and $B$
[Fig. 9(a), left]. Each unit is characterized by crease lengths $a_{x}, c_{x}$ and sector angles $\gamma_{x 1}, \gamma_{x 2}$. The subscript $x$ takes the values of $A$ or $B$, denoting the bottom unit $A$ or the top unit $B$, respectively. Without loss of generality, we assume $\gamma_{x 1}<$ $\gamma_{x 2}$. The following geometry constraints have to be satisfied to ensure the kinematic compatibility between the two units:

$$
a_{A}=a_{B}, \quad \frac{\cos \gamma_{A 1}}{\cos \gamma_{B 1}}=\frac{\cos \gamma_{A 2}}{\cos \gamma_{B 2}}=\frac{c_{B}}{c_{A}} .
$$

Folding of the stacked GFF structure is still a single-degreeof-freedom mechanism, and it can be described by the dihedral angle $\rho_{A 1}$ between two facets of the bottom unit $A$ [Fig. 9(a), right]. Alternatively, we can describe its folding by using the dihedral angles $\theta_{A i}$ (or $\left.\theta_{B i}\right)(i=1,2,3,4)$ between the facets of the bottom unit $A$ (or top unit $B$ ) and the reference $x-o-y$ plane. $\theta_{A i}$ and $\theta_{B i}$ are not independent of each other but satisfy the following constraints:

$$
\begin{aligned}
\cos \theta_{A 1} \tan \gamma_{A 1} & =\cos \theta_{B 1} \tan \gamma_{B 1}, \\
\sin \theta_{A 1} \sin \gamma_{A 1} & =\sin \theta_{A 2} \sin \gamma_{A 2} \\
& =\sin \theta_{A 3} \sin \gamma_{A 1}=\sin \theta_{A 4} \sin \gamma_{A 2}, \\
\sin \theta_{B 1} \sin \gamma_{B 1} & =\sin \theta_{B 2} \sin \gamma_{B 2} \\
& =\sin \theta_{B 3} \sin \gamma_{B 1}=\sin \theta_{B 4} \sin \gamma_{B 2} .
\end{aligned}
$$

When $\rho_{A 1}^{C 1} \leqslant \rho_{A 1}<180^{\circ}$, the structure is in nested-in configurations and $0^{\circ}<\theta_{A i} \leqslant 90^{\circ}$. In particular, when $\rho_{A 1}=$ $\rho_{A 1}^{C 1}$, the structure self-locks so that $\theta_{A 1}=\theta_{A 3}=90^{\circ}$ and $0^{\circ}<\theta_{A 2}=\theta_{A 4}<90^{\circ}$. On the other hand, when $180^{\circ} \leqslant$ $\rho_{A 1} \leqslant 360^{\circ}$, the structure is in bulged-out configurations and $-180^{\circ} \leqslant \theta_{A i} \leqslant 0^{\circ}$.

Here we focus on the bulged-out configuration of the stacked GFF structure. For $180^{\circ} \leqslant \rho_{A 1} \leqslant 360^{\circ}$, the folding can be divided into two stages. In the first stage, $\rho_{A 1}$ increases from $180^{\circ}$ to a critical value $\rho_{A 1}^{C 2}\left(\rho_{A 1}^{C 2}=\rho_{A 1}^{C 1}+90^{\circ}\right)$, and $\left|\theta_{A i}\right|$ increases accordingly from $0^{\circ}$ but remains smaller than $90^{\circ}$. The first stage ends when $\rho_{A 1}$ reaches a critical value $\rho_{A 1}{ }^{C 2}$. At this critical configuration, $\left|\theta_{A 1}\right|$ and $\left|\theta_{A 3}\right|$ reach $90^{\circ}$ simultaneously, while $\left|\theta_{A 2}\right|$ and $\left|\theta_{A 4}\right|$ are smaller than $90^{\circ}$. In the second stage of folding, $\rho_{A 1}$ continues increasing beyond $\rho_{A 1}{ }^{C 2}\left(\rho_{A 1}^{C 2}<\rho_{A 1} \leqslant 360^{\circ}\right)$, and $\left|\theta_{A 1}\right|$ and $\left|\theta_{A 3}\right|$ also continue increasing so that $\left|\theta_{A 1}\right|=\left|\theta_{A 3}\right|>90^{\circ}$; however, $\left|\theta_{A 2}\right|$ and $\left|\theta_{A 4}\right|$ decrease and remain smaller than $90^{\circ}\left(\left|\theta_{A 2}\right|=\left|\theta_{A 4}\right|<90^{\circ}\right)$. Details of the folding kinematics are discussed in [27]. Specifically, $\theta_{A i}(i=1,2,3,4)$ can be expressed as

$$
\begin{aligned}
& \theta_{A 1}=\theta_{A 3}=\left\{\begin{array}{ll}
\arcsin \frac{\sin \gamma_{A 2} \sin \rho_{A 1}}{\sqrt{\sin ^{2} \gamma_{A 1}+\sin ^{2} \gamma_{A 2}-2 \sin \gamma_{A 1} \sin \gamma_{A 2} \cos \rho_{A 1}}}, & 180^{\circ} \leqslant \rho_{A 1} \leqslant \rho_{A 1}^{C 2} \\
-\arcsin \frac{\sin \gamma_{A 2} \sin \rho_{A 1}}{\sqrt{\sin ^{2} \gamma_{A 1}+\sin ^{2} \gamma_{A 2}-2 \sin \gamma_{A 1} \sin \gamma_{A 2} \cos \rho_{A 1}}}-180^{\circ}, & \rho_{A 1}^{C 2}<\rho_{A 1} \leqslant 360^{\circ}
\end{array},\right. \\
& \theta_{A 2}=\theta_{A 4}=\arcsin \frac{\sin \gamma_{A 1} \sin \rho_{A 1}}{\sqrt{\sin ^{2} \gamma_{A 1}+\sin ^{2} \gamma_{A 2}-2 \sin \gamma_{A 1} \sin \gamma_{A 2} \cos \rho_{A 1}}}, \quad 180^{\circ} \leqslant \rho_{A 1} \leqslant 360^{\circ} .
\end{aligned}
$$

In the bulged-out configuration, the length $L$, width $W$, and height $H$ of the stacked GFF structure [Fig. 9(a), right] are given by

$$
\begin{aligned}
L & = \begin{cases}2 a_{A} \sin ((\xi+\eta) / 2), & 180^{\circ} \leqslant \rho_{A 1} \leqslant \rho_{A 1}^{C 2} \\
2 a_{A} \sin ((-\xi+\eta) / 2), & \rho_{A 1}^{C 2}<\rho_{A 1} \leqslant 360^{\circ}\end{cases} \\
W & = \begin{cases}2 c_{A} \sqrt{1-\sin ^{2} \gamma_{A 1} \sin ^{2} \theta_{A 1}} \cos [(\xi-\eta) / 2], & 180^{\circ} \leqslant \rho_{A 1} \leqslant \rho_{A 1}^{C 2}, \\
2 c_{A} \sqrt{1-\sin ^{2} \gamma_{A 1} \sin ^{2} \theta_{A 1}} \cos [(\xi+\eta) / 2], & \rho_{A 1}^{C 2}<\rho_{A 1} \leqslant 360^{\circ}\end{cases} \\
H_{A} & =c_{A} \sin \gamma_{A 1} \sin \left|\theta_{A 1}\right|, \quad H_{B}=c_{B} \sin \gamma_{B 1} \sin \theta_{B 1}, H=H_{A}+H_{B}, \quad 180^{\circ} \leqslant \rho_{A 1} \leqslant 360^{\circ},
\end{aligned}
$$


(a)
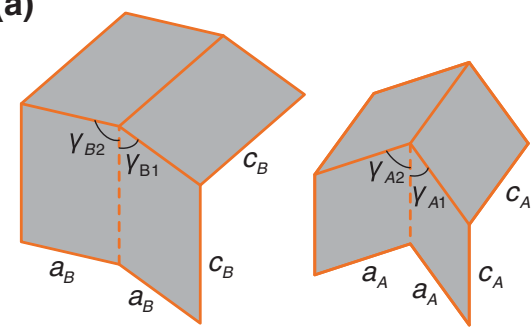

Top GFF unit A

Bottom GFF unit B Crease patterns

(b)

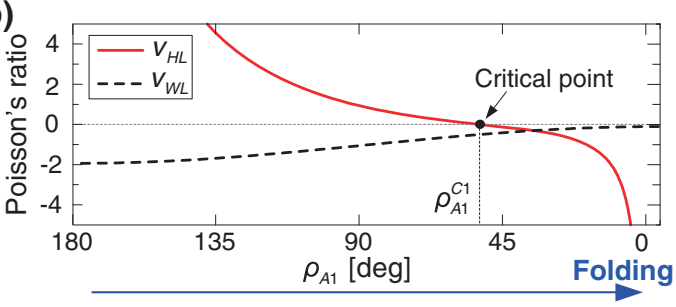

(c) 3D dilation based on a stacked GFF structure (bulged-out)

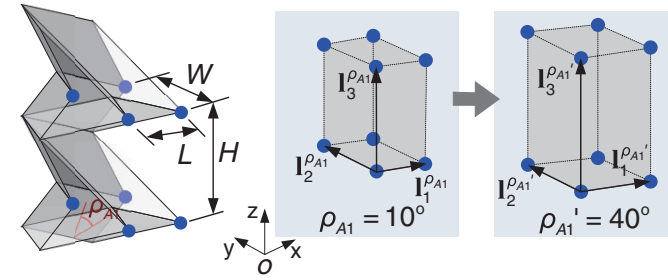

(e) 3D shear based on a

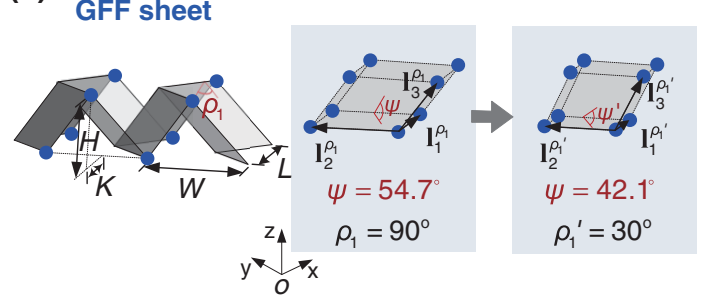

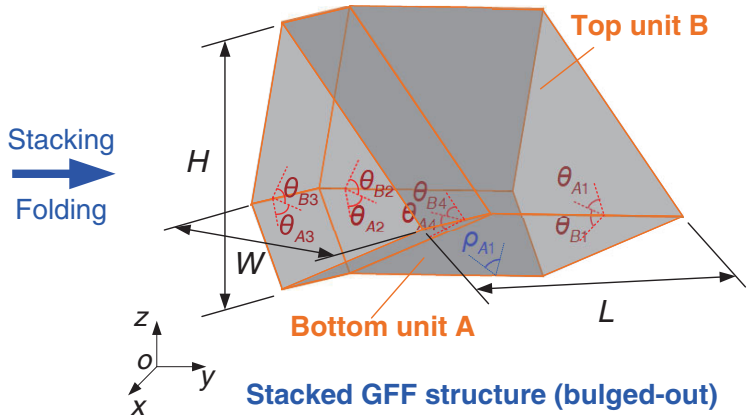

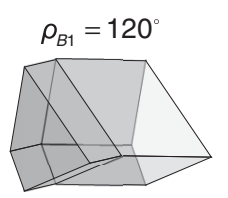

Before critical point

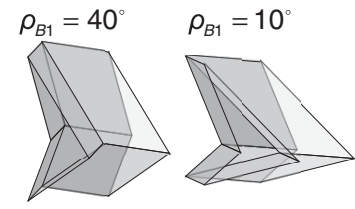

After critical point

Folding

(d) 3D Contraction and extension based on a stacked Miura-ori structure (bulged-out)

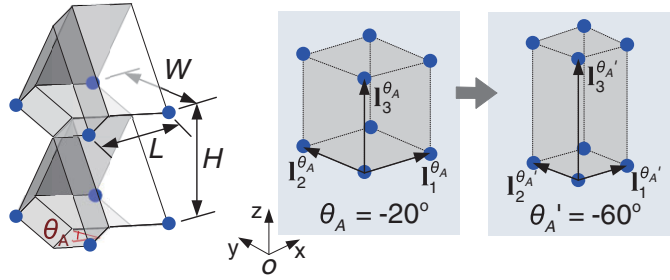

(f) 3D shuffle based on a

stacked Miura-ori structure (nested-in)

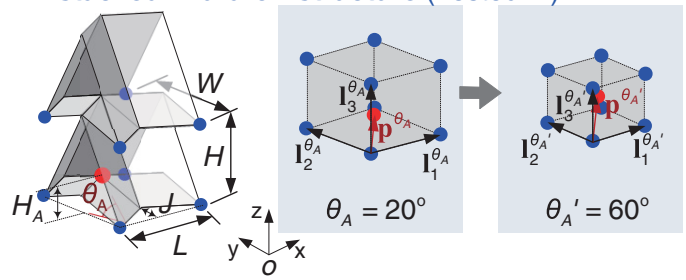

FIG. 9. Origami geometries and 3D diffusionless phase transformations. (a) Geometries of a stacked GFF structure (bulged out), where the constituent units' crease patterns (left) and the origami at a folded state (right) are given. (b) Poisson's ratios of the stacked GFF structure (bulged out). (c) 3D dilation achieved by transforming a lattice of vertex inclusions based on a stacked GFF structure (bulged out); (d) 3D contraction and extension achieved by transforming a lattice of vertex inclusions based on a stacked Miura-ori structure (bulged out); (e) 3D shear involved in transforming a lattice of vertex inclusions based on a GFF sheet; (f) 3D shuffle involved in transforming a lattice of vertex inclusions based on a stacked Miura-ori structure (nested in).

where $\xi$ and $\eta$ are similarly defined as Eq. (B3); i.e.,

$$
\begin{aligned}
& \xi=\arccos \frac{\cos \gamma_{A 1}}{\sqrt{1-\sin ^{2} \gamma_{A 1} \sin ^{2} \theta_{A 1}}}, \\
& \eta=\arccos \frac{\cos \gamma_{A 2}}{\sqrt{1-\sin ^{2} \gamma_{A 1} \sin ^{2} \theta_{A 1}}} .
\end{aligned}
$$

Based on the assignment of lattice points on the stacked GFF structure given in Fig. 9(c), the lattice vectors corresponding to two different configurations (with dihedral angle
$\rho_{A 1}$ and $\rho_{A 1}^{\prime}$ ) can be formulated as

$$
\begin{gathered}
\mathbf{l}_{1}^{\rho_{A 1}}=\left(\begin{array}{c}
\left.L\right|_{\rho_{A 1}} \\
0 \\
0
\end{array}\right), \quad \mathbf{l}_{2}^{\rho_{A 1}}=\left(\begin{array}{c}
0 \\
\left.W\right|_{\rho_{A 1}} \\
0
\end{array}\right), \quad \mathbf{l}_{3}^{\rho_{A 1}}=\left(\begin{array}{c}
0 \\
0 \\
\left.H\right|_{\rho_{A 1}}
\end{array}\right) ; \\
\mathbf{l}_{1}^{\rho_{A 1}^{\prime}}=\left(\begin{array}{c}
\left.L\right|_{\rho_{A 1}} \\
0 \\
0
\end{array}\right), \quad \mathbf{l}_{2}^{\rho_{A 1}^{\prime}}=\left(\begin{array}{c}
0 \\
\left.W\right|_{\rho_{A 1^{\prime}}} \\
0
\end{array}\right), \quad \mathbf{l}_{3}^{\rho_{A 1}^{\prime}}=\left(\begin{array}{c}
0 \\
0 \\
\left.H\right|_{\rho^{\prime}{ }_{A 1}}
\end{array}\right) .
\end{gathered}
$$

Since there is no diffusion, the folding-induced lattice transformation from angle $\rho_{A 1}$ to $\rho_{A 1}^{\prime}$ can be described by a 
transformation matrix $\mathbf{U}_{3 D-D}$ such that

$$
\left(\begin{array}{lll}
\mathbf{l}_{1}^{\rho_{A 1}^{\prime}} & \mathbf{l}_{2}^{\rho_{A 1}^{\prime}} & \mathbf{l}_{3}^{\rho_{A 1}^{\prime}}
\end{array}\right)^{T}=\mathbf{U}_{3 \mathrm{D}-\mathrm{D}}\left(\begin{array}{lll}
\mathbf{l}_{1}^{\rho_{A 1}} & \mathbf{l}_{2}^{\rho_{A 1}} & \mathbf{l}_{3}^{\rho_{A 1}}
\end{array}\right)^{T} .
$$

$\mathbf{U}_{3 D-D}$ (a diagonal matrix) describes the lattice-distortive strains that transform the lattice; the subscript "3D-D" means three-dimensional dilation. $\mathbf{U}_{3 D-D}$ can be written as

$$
\mathbf{U}_{3 \mathrm{D}-\mathrm{D}}=\left(\begin{array}{ccc}
\left.L\right|_{\rho_{A 1}^{\prime}} /\left.L\right|_{\rho_{A 1}} & 0 & 0 \\
0 & \left.W\right|_{\rho^{\prime}{ }_{A 1}} /\left.W\right|_{\rho_{A 1}} & 0 \\
0 & 0 & \left.H\right|_{\rho_{A 1}^{\prime}} /\left.H\right|_{\rho_{A 1}}
\end{array}\right) \text {. }
$$

By substituting the expressions of $L, W$, and $H$ [i.e., Eq. (C20)] into Eq. (C24), one can correlate $\mathbf{U}_{3 \mathrm{D}-\mathrm{D}}$ to the rigid-folding kinematics.

The observed 3D dilation has a close relation to the auxetic properties of the stacked GFF structure. Based on Eq. (C20), the Poisson's ratios can be calculated via

$$
\begin{aligned}
& v_{W L}=-\frac{\varepsilon_{W}}{\varepsilon_{L}}=-\frac{\mathrm{d} W / W}{\mathrm{~d} L / L}, \quad v_{H L}=-\frac{\varepsilon_{H}}{\varepsilon_{L}}=-\frac{\mathrm{d} H / H}{\mathrm{~d} L / L}, \\
& v_{W H}=-v_{W L} / v_{H L} .
\end{aligned}
$$

Figure 9(b) shows the Poisson's ratios $\left(v_{W L}\right.$ and $\left.v_{H L}\right)$ of the stacked GFF structure with respect to folding at the bulgedout configuration (i.e., $180^{\circ} \leqslant \rho_{A 1} \leqslant 360^{\circ}$ ). After the critical point $\rho_{A 1}^{C 2}$, the stacked GFF structure exhibits negative Poisson's ratios in all three directions. Therefore, in the folding range $\rho_{A 1}^{C 2}<\rho_{A 1} \leqslant 360^{\circ}$, if $\left.L\right|_{\rho_{A 1}^{\prime}}>\left.L\right|_{\rho_{A 1}}$, the negative Poisson's ratios indicate that $W_{\rho^{\prime} A 1}>\left.W\right|_{\rho_{A 1}}$ and $\left.H\right|_{\rho_{A 1}^{\prime}}>\left.H\right|_{\rho_{A 1}}$. This suggests that the lattice dilates along all of the three lattice vectors. Therefore, the tridirectional auxetic effect (i.e., negative Poisson's ratios in all three directions) is the root cause of the $3 \mathrm{D}$ dilation.

\section{3D Contraction and extension}

Three-dimensional contraction and extension is observed when transforming a 3D Bravais lattice of vertex inclusions based on a stacked Miura-ori structure in the bulged-out configuration $\left(-90^{\circ}<\theta_{A}<0\right)$ [27] [a kind of 3D Bravias lattice shown in Figs. 2(f) and 9(d)]. Construction of the stacked Miura-ori structure is introduced in Fig. 6(b), and its outer dimensions are given in Eq. (B7).

Based on the assignment of lattice points on the stacked Miura-ori structure given in Fig. 9(d), the lattice vectors corresponding to two different configurations (with folding angle $\theta_{A}$ and $\theta_{A}^{\prime}$ ) can be expressed as

$$
\begin{gathered}
\mathbf{l}_{1}^{\theta_{A}}=\left(\begin{array}{c}
\left.L\right|_{\theta_{A}} \\
0 \\
0
\end{array}\right), \quad \mathbf{l}_{2}^{\theta_{A}}=\left(\begin{array}{c}
0 \\
\left.W\right|_{\theta_{A}} \\
0
\end{array}\right), \quad \mathbf{l}_{3}^{\theta_{A}}=\left(\begin{array}{c}
0 \\
0 \\
\left.H\right|_{\theta_{A}}
\end{array}\right) ; \\
\mathbf{l}_{1}^{\theta_{A}^{\prime}}=\left(\begin{array}{c}
\left.L\right|_{\theta_{A^{\prime}}} \\
0 \\
0
\end{array}\right), \quad \mathbf{l}_{2}^{\theta^{\prime}{ }_{A}}=\left(\begin{array}{c}
0 \\
\left.W\right|_{\theta_{A^{\prime}}} \\
0
\end{array}\right), \quad \mathbf{l}_{3}^{\theta^{\prime}{ }_{A}}=\left(\begin{array}{c}
0 \\
0 \\
\left.H\right|_{\theta_{A^{\prime}}}
\end{array}\right) .
\end{gathered}
$$

Since there is no diffusion, the folding-induced lattice transformation from angle $\theta_{A}$ to $\theta_{A}^{\prime}$ can be described by a transformation matrix $\mathbf{U}_{3 \mathrm{D}-\mathrm{C} / \mathrm{E}}$ such that

$$
\left(\begin{array}{lll}
\mathbf{l}_{1}^{\theta_{A}^{\prime}} & \mathbf{l}_{2}^{\theta^{\prime}} \mathbf{l}_{3}^{\theta^{\prime}{ }_{A}}
\end{array}\right)^{T}=\mathbf{U}_{3 \mathrm{D}-\mathrm{D}}\left(\begin{array}{lll}
\mathbf{l}_{1}^{\theta_{A}} & \mathbf{l}_{2}^{\theta_{A}} & \mathbf{l}_{3}^{\theta_{A}}
\end{array}\right)^{T} .
$$

$\mathbf{U}_{3 \mathrm{D}-\mathrm{C} / \mathrm{E}}$ (a diagonal matrix) describes the lattice-distortive strains that transform the lattice; the subscript "3D-C/E" means three-dimensional contraction and extension. $\mathbf{U}_{3 \mathrm{D}-\mathrm{C} / \mathrm{E}}$ can be formulated as

$$
\mathbf{U}_{3 \mathrm{D}-\mathrm{C} / \mathrm{E}}=\left(\begin{array}{ccc}
\left.L\right|_{\theta^{\prime}{ }_{A}} /\left.L\right|_{\theta_{A}} & 0 & 0 \\
0 & \left.W\right|_{\theta^{\prime}{ }_{A}} /\left.W\right|_{\theta_{A}} & 0 \\
0 & 0 & \left.H\right|_{\theta^{\prime}{ }_{A}} /\left.H\right|_{\theta_{A}}
\end{array}\right) .
$$

By substituting the expressions of $L, W$, and $H$ [i.e., Eq. (B7)] into Eq. (C28), one can correlate $\mathbf{U}_{3 \mathrm{D}-\mathrm{C} / \mathrm{E}}$ to the rigid-folding kinematics.

The observed 3D contraction and extension has a close relation to the Poisson's ratios of the stacked Miura-ori structure (bulged-out configurations, with $-90^{\circ}<\theta_{A}<0$ ). Based on the outer dimensions given in Eq. (B7), the Poisson's ratios $v_{W L}$ and $v_{H L}$ have opposite signs in that

$$
\begin{aligned}
v_{W L} & =-\frac{\varepsilon_{W}}{\varepsilon_{L}}=-\frac{\mathrm{d} W}{\mathrm{~d} L} \frac{L}{W}=-\cos ^{2} \theta_{A} \tan ^{2} \gamma_{A}<0, \\
v_{H L} & =-\frac{\varepsilon_{H}}{\varepsilon_{L}}=-\frac{\mathrm{d} H}{\mathrm{~d} L} \frac{L}{H} \\
& =-\frac{\tan \gamma_{A}\left(1-\sin ^{2} \gamma_{A} \sin ^{2} \theta_{A}\right)}{\sin ^{2} \gamma_{A} \sin \theta_{A} \sqrt{\tan ^{2} \gamma_{B}-\cos ^{2} \theta_{A} \tan ^{2} \gamma_{A}}}>0 .
\end{aligned}
$$

Hence, in the folding range $-90^{\circ}<\theta_{A}<0$, if $\left.L\right|_{\theta^{\prime} A}<\left.L\right|_{\theta_{A}}$, the negative Poisson's ratios $v_{W L}$ indicates that $W_{\theta^{\prime}}<\left.W\right|_{\theta_{A}}$; however, the positive Poisson's ratio $v_{H L}$ suggests that $\left.H\right|_{\theta^{\prime} A}>\left.H\right|_{\theta_{A}}$. As a result, the lattice contracts in the $L$ and $W$ directions but extends in the $H$ direction. In other words, the opposite Poisson's ratios are the origin of the $3 \mathrm{D}$ contraction and extension.

\section{3D Shear}

Three-dimensional shear is observed when transforming a 3D Bravais lattice of vertex inclusions based on a GFF sheet [27] [a kind of 3D Bravias lattice shown in Figs. 2(g) and 9(e)]. A GFF unit is characterized by crease lengths $a, c$ and sector angles $\gamma_{1}, \gamma_{2}$. Without the loss of generality, we assume $\gamma_{1}<\gamma_{2}$. Its folding can be described by the dihedral angle $\rho_{1}$ or the folding angles $\theta_{i}(i=1,2,3,4)$ (defined as the dihedral angle between the facets and the reference $x$-o-y plane). Folding of a GFF unit can also be divided into two stages. In the first stage, $\rho_{1}$ decreases from $180^{\circ}$ to a critical value $\rho_{1}^{C}$, while $\theta_{i}(i=1,2,3,4)$ increase from $0^{\circ}$ but remains smaller than $90^{\circ}$. At the end of the first stage, $\rho_{1}$ reaches a critical value $\rho_{1}^{C}$, and $\theta_{1}$ and $\theta_{3}$ reach $90^{\circ}$, while $\theta_{2}$ and $\theta_{4}$ remain smaller than $90^{\circ}$. In the second stage, $\rho_{1}$ continues decreasing towards 0 , and $\theta_{1}$ and $\theta_{3}$ keep increasing $\left(\theta_{1}=\theta_{3}>90^{\circ}\right)$, but $\theta_{2}$ and $\theta_{4}$ start to decrease and remain $\theta_{2}=\theta_{4}<90^{\circ}$. Details of the folding kinematics are discussed in [27]. Specifically, 
$\theta_{i}(i=1,2,3,4)$ can be expressed as

$$
\begin{aligned}
& \theta_{1}=\theta_{3}=\left\{\begin{array}{ll}
\arcsin \frac{\sin \gamma_{2} \sin \rho_{1}}{\sqrt{\sin ^{2} \gamma_{1}+\sin ^{2} \gamma_{2}-2 \sin \gamma_{1} \sin \gamma_{2} \cos \rho_{1}}}, & \rho_{1}{ }^{C} \leqslant \rho_{1} \leqslant 180^{\circ} \\
180^{\circ}-\arcsin \frac{\sin \gamma_{2} \sin \rho_{1}}{\sqrt{\sin ^{2} \gamma_{1}+\sin ^{2} \gamma_{2}-2 \sin \gamma_{1} \sin \gamma_{2} \cos \rho_{1}}}, & 0 \leqslant \rho_{1}<\rho_{1}{ }^{C}
\end{array},\right. \\
& \theta_{2}=\theta_{4}=\arcsin \frac{\sin \gamma_{1} \sin \rho_{1}}{\sqrt{\sin ^{2} \gamma_{1}+\sin ^{2} \gamma_{2}-2 \sin \gamma_{1} \sin \gamma_{2} \cos \rho_{1}}}, \quad 0 \leqslant \rho_{1} \leqslant 180^{\circ} .
\end{aligned}
$$

The outer dimensions of a GFF unit are

$$
\begin{aligned}
& L= \begin{cases}2 a \sin [(\xi+\eta) / 2], & \rho_{1}{ }^{C} \leqslant \rho_{1} \leqslant 180, \\
2 a \sin [(-\xi+\eta) / 2], & 0 \leqslant \rho_{1}<\rho_{1}{ }^{C}\end{cases} \\
& W= \begin{cases}2 c \sqrt{1-\sin ^{2} \gamma_{1} \sin ^{2} \theta_{1}} \cos [(\xi-\eta) / 2], & \rho_{1}{ }^{C} \leqslant \rho_{1} \leqslant 180, \\
2 c \sqrt{1-\sin ^{2} \gamma_{1} \sin ^{2} \theta_{1}} \cos [(\xi+\eta) / 2], & 0 \leqslant \rho_{1}<\rho_{1}{ }^{C},\end{cases} \\
& H=c \sin \gamma_{1} \sin \theta_{1}, \quad K=\sqrt{a^{2}-H^{2}-(W / 2)^{2}}, \quad 0 \leqslant \rho_{1} \leqslant 180,
\end{aligned}
$$

where $\xi$ and $\eta$ are similarly defined as Eq. (A1); i.e.,

$$
\xi=\arccos \frac{\cos \gamma_{1}}{\sqrt{1-\sin ^{2} \gamma_{1} \sin ^{2} \theta_{1}}}, \quad \eta=\arccos \frac{\cos \gamma_{2}}{\sqrt{1-\sin ^{2} \gamma_{1} \sin ^{2} \theta_{1}}} .
$$

Based on the assignment of lattice points on the GFF sheet given in Fig. 9(e), the lattice vectors corresponding to two different configurations (with dihedral angles $\rho_{1}$ and $\rho_{1}^{\prime}$ ) are

$$
\begin{aligned}
& \mathbf{l}_{1}^{\rho_{1}}=\left(\begin{array}{c}
\left.L\right|_{\rho_{1}} \\
0 \\
0
\end{array}\right), \quad \mathbf{l}_{2}^{\rho_{1}}=\left(\begin{array}{c}
0 \\
\left.W\right|_{\rho_{1}} \\
0
\end{array}\right), \quad \mathbf{l}_{3}^{\rho_{1}}=\left(\begin{array}{c}
-\left.K\right|_{\rho_{1}} \\
-\left.W\right|_{\rho_{1}} / 2 \\
\left.H\right|_{\rho_{1}}
\end{array}\right) ; \\
& \mathbf{l}_{1}^{\rho^{\rho^{\prime}}{ }_{1}}=\left(\begin{array}{c}
\left.L\right|_{\theta_{1}{ }^{\prime}} \\
0 \\
0
\end{array}\right), \quad \mathbf{l}_{2}^{\rho^{\prime}{ }_{1}}=\left(\begin{array}{c}
0 \\
\left.W\right|_{\theta_{1}{ }^{\prime}} \\
0
\end{array}\right), \quad \mathbf{l}_{3}^{\rho^{\prime}{ }_{1}}=\left(\begin{array}{c}
-\left.K\right|_{\rho^{\prime}{ }_{1}} \\
-\left.W\right|_{\rho^{\prime}{ }_{1}} / 2 \\
\left.H\right|_{\rho^{\prime}{ }_{1}}
\end{array}\right) .
\end{aligned}
$$

Since there is no diffusion, the folding-induced lattice transformation from angle $\rho_{1}$ to $\rho_{1}^{\prime}$ can be described by a transformation matrix $\mathbf{U}_{3 D-S}$ such that

$$
\left(\begin{array}{lll}
\mathbf{l}_{1}^{\rho_{1}^{\prime}} & \mathbf{l}_{2}^{\rho^{\prime}{ }_{1}} & \mathbf{l}_{3}^{\rho^{\prime}{ }_{1}}
\end{array}\right)^{T}=\mathbf{U}_{3 \mathrm{D}-\mathrm{S}}\left(\begin{array}{lll}
\mathbf{l}_{1}^{\rho_{1}} & \mathbf{l}_{2}^{\rho_{1}} & \mathbf{l}_{3}^{\rho_{1}}
\end{array}\right)^{T}
$$

$\mathbf{U}_{3 \mathrm{D}-\mathrm{S}}$ describes the lattice-distortive strains that transform the lattice; the subscript "3D-S" indicates three-dimensional shear. $\mathbf{U}_{3 \mathrm{D}-\mathrm{S}}$ can be formulated as

$$
\mathbf{U}_{3 \mathrm{D}-\mathrm{S}}=\left(\begin{array}{ccc}
\left.L\right|_{\rho^{\prime}{ }_{1}} /\left.L\right|_{\rho_{1}} & 0 & 0 \\
0 & \left.W\right|_{\rho_{\rho_{1}}} /\left.W\right|_{\rho_{1}} & 0 \\
\frac{\left.K\right|_{\rho_{1}}\left(\left.H\right|_{\rho^{\prime}{ }_{1}} /\left.H\right|_{\rho_{1}}\right)-\left.K\right|_{\rho^{\prime} 1}}{\left.L\right|_{\rho_{1}}} & \frac{\left.W\right|_{\rho_{1}}\left(\left.H\right|_{\rho^{\prime}{ }_{1}} /\left.H\right|_{\rho_{1}}\right)-\left.W\right|_{\rho^{\prime} 1}}{\left.2 W\right|_{\rho_{1}}} & \left.H\right|_{\rho_{\rho_{1}} S} /\left.H\right|_{\rho_{1}}
\end{array}\right) .
$$

By substituting the expressions of $L, W, H$, and $K$ [i.e., Eq. (C31)] into Eq. (C35), one can correlate $\mathbf{U}_{3 \mathrm{D}-\mathrm{S}}$ to the rigid-folding kinematics.

$\mathbf{U}_{3 D-S}$ is not a diagonal matrix. Its nontrivial main diagonal elements describe the contraction and (or) extension along the lattice vectors; and its nonzero off-diagonal elements describe the distortion due to shear. Note that the GFF origami is characterized by its ability in achieving outof-plane shearing deformation during folding, which manifests as the changes of the angle $\psi$ [27]. For example, by folding the GFF unit $\left(a=c, \gamma_{1}=36^{\circ}, \gamma_{2}=72^{\circ}\right)$ from $\rho_{1}=90^{\circ}$ to $\rho_{1}=30^{\circ}$, the angle $\psi$ changes from $54.7^{\circ}$ to $42.1^{\circ}$. As a result, the out-of-plane shearing deformation is the root cause of the $3 \mathrm{D}$ shear component in lattice transformations.

\section{3D Shuffle}

Three-dimensional shuffle is observed when transforming a 3D non-Bravais lattice of vertex inclusions based on a stacked Miura-ori structure in the nested-in configurations $\left(0 \leqslant \theta_{A} \leqslant 90^{\circ}\right)$, shown in Figs. 2(h) and 9(f). Construction of the stacked Miura-ori structure has been introduced in Fig. 6(b). Its folding can be described by the dihedral folding angle $\theta_{A}$ between a facet of the bottom Miura-ori unit and the reference $x-o-y$ plane. In the nested-in configurations, the 
TABLE IV. Correlations between diffusionless lattice transformations and origami kinematic properties.

\begin{tabular}{|c|c|c|c|}
\hline $2 \mathrm{D}$ and $3 \mathrm{D}$ & $\begin{array}{l}\text { Diffusionless lattice } \\
\text { transformations }\end{array}$ & Origami kinematic properties & $\begin{array}{c}\text { Representative } \\
\text { origami structures }\end{array}$ \\
\hline \multirow{4}{*}{$2 \mathrm{D}$} & Dilation & Negative in-plane Poisson's ratio & Miura-ori sheet \\
\hline & Contraction and extension & Positive in-plane Poisson's ratio & Egg-box sheet \\
\hline & Shear & In-plane shearing deformation mechanism & SC origami sheet \\
\hline & Shuffle & $\begin{array}{l}\text { Changes in the relative positions among } \\
\text { characteristic entities }\end{array}$ & Miura-ori sheet \\
\hline \multirow{4}{*}{$3 \mathrm{D}$} & Dilation & $\begin{array}{l}\text { Tri-directional auxetic effect } \\
\quad \text { (negative Poisson's ratios in three directions) }\end{array}$ & $\begin{array}{c}\text { Stacked Miura-ori } \\
\text { structure (bulged out) }\end{array}$ \\
\hline & Contraction and extension & Opposite Poisson's ratios & GFF sheet \\
\hline & Shear & $\begin{array}{l}\text { Out-of-plane shearing deformation } \\
\text { mechanism }\end{array}$ & $\begin{array}{l}\text { Stacked Miura-ori } \\
\text { structure (nested in) }\end{array}$ \\
\hline & Shuffle & $\begin{array}{l}\text { Changes in the relative positions among } \\
\text { characteristic entities }\end{array}$ & $\begin{array}{l}\text { Stacked Miura-ori } \\
\text { structure (bulged out) }\end{array}$ \\
\hline
\end{tabular}

structure's outer dimensions are

$$
\begin{aligned}
H_{A} & =c_{A} \sin \gamma_{A} \sin \theta_{A}, \quad H_{B}=c_{B} \sin \gamma_{B} \sin \theta_{B}, \\
H & =H_{B}-H_{A}, \quad L=2 a_{A} \frac{\cos \theta_{A} \sin \gamma_{A}}{\sqrt{1-\sin ^{2} \gamma_{A} \sin ^{2} \theta_{A}}}, \\
J & =\frac{a_{A}}{\sqrt{1+\tan ^{2} \gamma_{A} \cos ^{2} \theta_{A}}}, \quad W=2 c_{A} \sqrt{1-\sin ^{2} \gamma_{A} \sin ^{2} \theta_{A}} .
\end{aligned}
$$

Unlike the three aforementioned cases, here, three lattice vectors are not sufficient to describe the $3 \mathrm{D}$ non-Bravais lattice here due to the additional lattice point inside the unit cell. Therefore, a shift vector $\mathbf{p}$ has to be incorporated in addition to the lattice vectors $\mathbf{l}_{\mathbf{i}}$ [43], similar to the $2 \mathrm{D}$ shuffle scenario. The lattice vectors $\mathbf{l}_{\mathbf{i}}$ describe the constituent Bravais lattice, and the shift vector $\mathbf{p}$ describes the offset between the two congruent lattices. Specifically, these vectors corresponding to two different configurations (with folding angle $\theta_{A}$ and $\theta_{A}^{\prime}$ ) can be formulated as

$$
\begin{aligned}
& \mathbf{l}_{1}^{\theta}=\left(\begin{array}{c}
\left.L\right|_{\theta} \\
0 \\
0
\end{array}\right), \quad \mathbf{l}_{2}^{\theta}=\left(\begin{array}{c}
0 \\
\left.W\right|_{\theta} \\
0
\end{array}\right), \\
& \mathbf{l}_{3}^{\theta}=\left(\begin{array}{c}
0 \\
0 \\
\left.H\right|_{\theta}
\end{array}\right), \quad \mathbf{p}^{\theta}=\left(\begin{array}{c}
\left.L\right|_{\theta} / 2 \\
\left.J\right|_{\theta}+\left.W\right|_{\theta} / 2 \\
\left.H\right|_{\theta}
\end{array}\right) ; \\
& \mathbf{l}_{1}^{\theta^{\prime}}=\left(\begin{array}{c}
\left.L\right|_{\theta^{\prime}} \\
0 \\
0
\end{array}\right), \quad \mathbf{l}_{2}^{\theta^{\prime}}=\left(\begin{array}{c}
0 \\
\left.W\right|_{\theta^{\prime}} \\
0
\end{array}\right), \\
& \mathbf{l}_{3}^{\theta}=\left(\begin{array}{c}
0 \\
0 \\
\left.H\right|_{\theta^{\prime}}
\end{array}\right), \quad \mathbf{p}^{\theta^{\prime}}=\left(\begin{array}{c}
\left.L\right|_{\theta^{\prime}} / 2 \\
\left.J\right|_{\theta^{\prime}}+\left.W\right|_{\theta^{\prime}} / 2 \\
\left.H_{A}\right|_{\theta^{\prime}}
\end{array}\right) .
\end{aligned}
$$

Since there is no diffusion, the folding-induced lattice transformation from angle $\theta$ to $\theta^{\prime}$ can be described as a combination of deformation and shift. Hence, we can formulate the transformation matrix $\mathbf{T}_{\text {3D-SH }}$ such that

$$
\left(\begin{array}{llll}
\mathbf{l}_{1}^{\theta^{\prime}} & \mathbf{l}_{2}^{\theta^{\prime}} & \mathbf{l}_{3}^{\theta^{\prime}} & \mathbf{p}^{\theta^{\prime}}
\end{array}\right)^{T}=\mathbf{T}_{3 \mathrm{D}-\mathrm{SH}}\left(\begin{array}{llll}
\mathbf{l}_{1}^{\theta} & \mathbf{l}_{2}^{\theta} & \mathbf{l}_{3}^{\theta} & \mathbf{p}^{\theta}
\end{array}\right)^{T} .
$$

Here the subscript "3D-SH" means three-dimensional shuffle, and $\mathbf{T}_{3 \mathrm{D}-\mathrm{SH}}$ can be written as

$$
\mathbf{T}_{2 \mathrm{D}-\mathrm{SH}}=\left(\begin{array}{ccccc} 
& & & \\
& \mathbf{U}_{3 \mathrm{D}-\mathrm{D}} & & 0 \\
& & & 0 \\
\mu_{1} & \mu_{2} & \mu_{3} & \lambda
\end{array}\right) .
$$

$\mathbf{T}_{3 \mathrm{D}-\mathrm{SH}}$ is not a diagonal matrix, and it reflects how the lattice-distortive strain and shuffle component are integrated in the transformation. The submatrix $\mathbf{U}_{3 \mathrm{D}-\mathrm{D}}$ relates to the $3 \mathrm{D}$ dilation, which describes the lattice-distortive strains of the constituent 3D Bravias lattice. It can be replaced by other $3 \mathrm{D}$ transformation matrices (i.e., $\mathbf{U}_{3 \mathrm{D}-\mathrm{S}}, \mathbf{U}_{3 \mathrm{D}-\mathrm{C} / \mathrm{E}}$, or $\mathbf{U}_{3 \mathrm{D}-\mathrm{S}}$ ) if different origami structures are used. The nontrivial elements in the fourth row quantify the $3 \mathrm{D}$ shuffle component; they satisfy the following relationship:

$$
\left(\mathbf{p}^{\theta^{\prime}}\right)^{T}=\left(\begin{array}{llll}
\mu_{1} & \mu_{2} & \mu_{3} & \lambda
\end{array}\right)\left(\begin{array}{llll}
\mathbf{l}_{1}^{\theta} & \mathbf{l}_{2}^{\theta} & \mathbf{l}_{3}^{\theta} & \mathbf{p}^{\theta}
\end{array}\right)^{T} .
$$

By substituting the expressions of $L, W, H, H_{A}$, and $J$ [i.e., Eq. (C36)] into Eqs. (C39) and (C40), one can correlate $\mathbf{T}_{3 \mathrm{D}-\mathrm{SH}}$ to the rigid-folding kinematics.

Again, we emphasize that changes in the relative positions among vertices (or other characteristic entities) are ubiquitous during origami folding, and it is the fundamental mechanism that generates the shuffle during lattice transformation.

Overall, this section examines all types of 2D and 3D diffusionless lattice transformations by formulating the lattice transformation matrices based on the rigid-folding kinematics. Although the analyses here are based on specific examples of origami structures, the uncovered correlations between the diffusionless lattice transformations and the kinematic properties of origami structures (summarized in Table IV) hold strong generality that can be used to guide the design and control of transformable lattice systems. 
[1] K. Bhattacharya, S. Conti, G. Zanzotto, and J. Zimmer, Nature 428, 55 (2004).

[2] K. Bhattacharya, Microstructure of Martensite: Why It Forms and How It Gives Rise to the Shape-Memory Effect (Oxford University Press, New York, 2004).

[3] B. Haghpanah, H. Ebrahimi, D. Mousanezhad, J. Hopkins, and A. Vaziri, Adv. Eng. Mater. 18, 643 (2016).

[4] Y. Tian, Y. Zhang, T. Wang, H. L. Xin, H. Li, and O. Gang, Nat. Mater. 15, 654 (2016).

[5] X. Zheng, W. Smith, J. Jackson, B. Moran, H. Cui, D. Chen, J. Ye, N. Fang, N. Rodriguez, T. Weisgraber, and C. M. Spadaccini, Nat. Mater. 15, 1100 (2016).

[6] C. Coulais, E. Teomy, K. de Reus, Y. Shokef, and M. van Hecke, Nature 535, 529 (2016).

[7] N. I. Zheludev and Y. S. Kivshar, Nat. Mater. 11, 917 (2012).

[8] T. A. Schaedler, A. J. Jacobsen, A. Torrents, A. E. Sorensen, J. Lian, J. R. Greer, L. Valdevit, and W. B. Carter, Science 334, 962 (2011).

[9] X. Zheng, H. Lee, T. H. Weisgraber, M. Shusteff, J. DeOtte, E. B. Duoss, J. D. Kuntz, M. M. Biener, Q. Ge, J. A. Jackson, S. O. Kucheyev, N. X. Fang, and C. M. Spadaccini, Science 344, 1373 (2014).

[10] J. B. Berger, H. N. G. Wadley, and R. M. McMeeking, Nature 543, 533 (2017).

[11] D. Z. Rocklin, S. Zhou, K. Sun, and X. Mao, Nat. Commun. 8, 14201 (2017).

[12] M. V. Rybin, D. S. Filonov, K. B. Samusev, P. A. Belov, Y. S. Kivshar, and M. F. Limonov, Nat. Commun. 6, 10102 (2015).

[13] T. Bückmann, M. Kadic, R. Schittny, and M. Wegener, Proc. Natl. Acad. Sci. USA 112, 4930 (2015).

[14] J. A. Faber, A. F. Arrieta, and A. R. Studart, Science 359, 1386 (2018).

[15] B. Liu, J. L. Silverberg, A. A. Evans, C. D. Santangelo, R. J. Lang, T. C. Hull, and I. Cohen, Nat. Phys. 14, 811 (2018).

[16] A. Lebée, Int. J. Space Struct. 30, 55 (2015).

[17] D. Rus and M. T. Tolley, Nat. Rev. Mater. 3, 101 (2018).

[18] L. H. Dudte, E. Vouga, T. Tachi, and L. Mahadevan, Nat. Mater. 15, 583 (2016).

[19] Y. Chen, R. Peng, and Z. You, Science 349, 396 (2015).

[20] S. J. P. Callens and A. A. Zadpoor, Mater. Today 21, 241 (2018).

[21] Z. Zhai, Y. Wang, and H. Jiang, Proc. Natl. Acad. Sci. USA 115, 2032 (2018).

[22] M. Schenk and S. D. Guest, Proc. Natl. Acad. Sci. USA 110, 3276 (2013).
[23] K. Fuchi, J. Tang, S. Member, B. Crowgey, S. Member, A. R. Diaz, E. J. Rothwell, and R. O. Ouedraogo, IEEE Antennas Wireless Propag. Lett. 11, 473 (2012).

[24] M. Thota, S. Li, and K. W. Wang, Phys. Rev. B 95, 064307 (2017).

[25] M. Thota and K. W. Wang, J. Appl. Phys. 122, 154901 (2017).

[26] S. Waitukaitis and M. van Hecke, Phys. Rev. E 93, 023003 (2016).

[27] H. Fang, S. Li, H. Ji, and K. W. Wang, Phys. Rev. E 94, 043002 (2016).

[28] L. Delaey, in Phase Transformations in Materials, edited by G. Kostorz (Wiley-VCH, Weinheim, Germany, 2001), pp. 583-654.

[29] M. Schenk, Folded shell structures, Ph.D. thesis, University of Cambridge, 2011.

[30] M. Cohen, G. B. Olson, and P. C. Clapp, in Proceedings of the International Conference on Martensitic Transformations (ICOMAT 1979) (Cambridge, MA, 1979), pp. 1-11 (unpublished).

[31] See Supplemental Material at http://link.aps.org/supplemental/ 10.1103/PhysRevResearch.1.023010 for other examples of discrete symmetry switches, the definition and quantification methods of continuous symmetry measures, and a detailed application example.

[32] H. Zabrodsky, S. Peleg, and D. Avnir, J. Am. Chem. Soc. 114, 7843 (1992).

[33] H. Zabrodsky and D. Avnir, Adv. Mol. Struct. Res. 1, 1 (1995).

[34] H. Zabrodsky, S. Peleg, and D. Avnir, J. Am. Chem. Soc. 115, 8278 (1993).

[35] T. Frenzel, M. Kadic, and M. Wegener, Science 358, 1072 (2017).

[36] L. M. Nash, D. Kleckner, A. Read, V. Vitelli, A. M. Turner, and W. T. M. Irvine, Proc. Natl. Acad. Sci. USA 112, 14495 (2015).

[37] G. Ma and P. Sheng, Sci. Adv. 2, e1501595 (2016).

[38] J. Y. Ou, E. Plum, L. Jiang, and N. I. Zheludev, Nano Lett. 11, 2142 (2011).

[39] A. E. Marras, L. Zhou, H.-J. Su, and C. E. Castro, Proc. Natl. Acad. Sci. USA 112, 713 (2015).

[40] M. Thota and K. W. Wang, J. Sound Vibr. 430, 93 (2018).

[41] J. L. Silverberg, A. A. Evans, L. McLeod, R. C. Hayward, T. Hull, C. D. Santangelo, and I. Cohen, Science 345, 647 (2014).

[42] C. Kittel, Introduction to Solid State Physics, 8th ed. (Wiley, New York, 2004).

[43] M. Pitteri, J. Elasticity 15, 3 (1985). 